\title{
Building arbitrage-free implied volatility: Sinkhorn's algorithm and variants
}

\author{
Hadrien De March ${ }^{\nexists 2}$ and Pierre Henry-Labordère甲 \\ ${ }^{1}$ École Polytechnique, CMAP \\ ${ }^{2}$ QantEv, Research Team \\ ${ }^{3}$ Société Générale, Global Market Quantitative Research
}

December 8, 2020

\begin{abstract}
We consider the classical problem of building an arbitrage-free implied volatility surface from bid-ask quotes. We design a fast numerical procedure, for which we prove the convergence, based on the Sinkhorn algorithm that has been recently used to solve efficiently (martingale) optimal transport problems.
\end{abstract}

\section{INTRODUCTION}

Building arbitrage-free implied volatility surfaces from bid-ask quotes is a long-standing issue. In particular, this is needed for market-makers in equity Vanillas. This is also needed for pricing exotic options when using risk-neutral models calibrated to Vanillas, as for the local volatility model [10] or for local stochastic volatility models [20]. In this purpose, various approaches have been considered. We review in the next section some of them and highlight their main drawbacks. A good method should be able to:

(1) produce calendar/butterfly arbitrage-free surfaces.

(2) fit market quotes perfectly within bid/ask spreads.

(3) fit smiles before earnings (with Mexican hat-shape curves).

(4) fit quickly.

1.1. Review of literature. In the following, for ease of notations, we assume zero rates/dividends (see however remark (2.4) for explanations how to include exactly cash/yield dividends (and deterministic rates) in this framework). For completeness, we recall that the market price of a call option $\mathcal{C}(T, K) \in\left[\left(S_{0}-K\right)_{+}, S_{0}\right]$ with maturity $T$ and strike $K$ is quoted in terms of an implied volatility $\sigma_{\mathrm{BS}}(T, K)$ defined as the constant volatility $\sigma_{\mathrm{BS}}(T, K):=\sigma$ such that $\mathcal{C}(T, K)=\operatorname{BS}\left(S_{0}, K, \sigma \sqrt{T}\right)$ where $S_{0}$ is the spot price value at $t=0$ and BS denotes the Black-Scholes formula:

$$
\operatorname{BS}\left(S_{0}, K, \omega\right):=S_{0} N\left(d_{+}\right)-K N\left(d_{-}\right) .
$$

*hadrien.de-march@polytechnique.org

${ }^{\dagger}$ pierre.henry-labordere@sgcib.com 
Here $d_{ \pm}=\frac{\ln \left(\frac{S_{0}}{K}\right)}{\omega} \pm \frac{\omega}{2}$ and $N(x)$ is the standard normal cumulative distribution function. As $\mathrm{BS} \in\left[\left(S_{0}-K\right)_{+}, S_{0}\right]$ is strictly increasing in $\omega$, the implied volatility is unique.

1.1.1. SVM-based parameterization. We consider the implied volatility associated to a stochastic volatility model (in short SVM), depending on some parameters: initial volatility, spot-volatility correlation, volatility-of-volatility, etc.... For example, one can consider an SVM, defined by an homogeneous Itô diffusion:

$$
d S_{t}=C\left(S_{t}\right) a_{t} d W_{t}, \quad d a_{t}=(\cdots) d t+\sigma\left(a_{t}\right) d Z_{t}, \quad d\langle Z, W\rangle_{t}=\rho d t
$$

As coming from a risk-neural model (i.e., $S$. is a (local) martingale - see [15] for sufficient and necessary conditions on the coefficients of the diffusion with $C(s):=s$ for imposing that $S$ is not only a local martingale but a true martingale), the resulting implied volatility,$\sigma_{\mathrm{BS}}(\cdot, \cdot)$, for which $\mathbb{E}\left[\left(S_{T}-K\right)_{+}\right]=\mathrm{BS}\left(S_{0}, K, \sigma_{\mathrm{BS}}(T, K) \sqrt{T}\right)$, is arbitrage-free. In practice, the implied volatility can not be derived in closed-form and therefore the calibration of the parameters of the SVM on market prices can be quite time-consuming. In order to speed up this optimization, one can rely on the approximation of the implied volatility in the short-maturity regime. At the first-order in the maturity $T$, one can derive a generic formula [15], obtained by using short-time asymptotics of the heat kernel on Cartan-Hadamard manifolds, for which the cut-locus is empty:

$$
\begin{aligned}
& \sigma_{\mathrm{BS}}(T, K) \underset{T \rightarrow 0}{\sim} \frac{\ln \frac{K}{S_{0}}}{\int_{S_{0}}^{K} \frac{d x}{a^{*}(x)}}\left(1+a_{1}(K) T+O\left(T^{2}\right)\right), \\
& a^{*}(x):=\operatorname{argmin}_{a} d_{\text {geo }}\left(x, a \mid S_{0}, a_{0}\right),
\end{aligned}
$$

where the geodesic distance $d_{\text {geo }}$ is

$$
d_{\text {geo }}\left(y_{2}, x_{2} \mid y_{1}, x_{1}\right):=\int_{y_{1}}^{y_{2}} \frac{F\left(y^{\prime}\right)}{\sqrt{F\left(y^{\prime}\right)-C^{2}}} d y^{\prime},
$$

with $C$ defined by the equation $x_{2}-x_{1}=\int_{y_{1}}^{y_{2}} \frac{C}{\sqrt{F\left(y^{\prime}\right)-C^{2}}} d y^{\prime}$, and $F(y):=\frac{2}{a(y)^{2}\left(1-\rho^{2}\right)}$, with the new coordinates $x:=\int_{S_{0}}^{S} \frac{d z}{C(z)}-\rho \int_{a_{0}}^{a} \frac{u}{\sigma(u)} d u$ and $y:=\sqrt{1-\rho^{2}} \int_{a_{0}}^{a} \frac{u}{\sigma(u)} d u$. The lengthly expression for $a_{1}(K)$ is not reported and can be found in [15]. As an example, one can cite the SABR parameterization for which $C(S):=S^{\beta}$ with $\beta \in[0,1)$ and $a_{t}$ is a log-normal process. The resulting manifold is the $2 d$ hyperbolic space $\mathbb{H}^{2}$. Let us remark that similar formulas can be also derived using large deviations (see [11] for extensive references).

By construction, the implied volatility is arbitrage-free in strike as the parametrization comes from a risk-neutral model. However, the maturity $T$ should be "small" in order to preserve the validity of our approximate formula (1.1). The arbitrability in maturity is not ensured as the calibration is performed by considering separately each time slice. Moreover, as our formula depends on a finite number of parameters, it is not possible to match exactly market prices. From a numerical point of view, the calibration involves a non-convex optimization, which is not guaranteed to converge. This solution only solves (4) and partially (1). 
1.1.2. Parametric form. Another approach is to start directly with a parametrization of the implied volatility. As an example, commonly used by practitioners, we have the SVI parametrization [13.

$$
\sigma_{B S}(T, K)=a+b\left(\rho(k-m)+\sqrt{(k-m)^{2}+\sigma^{2}}\right),
$$

depending on five parameters $a, b, \rho, m$ and $\sigma$. Note that this parametrization can be linked with the large maturity limit of the implied volatility in the Heston model. Despite its simplicity, the arbitrage-freeness in strike and maturity is not guaranteed, see however [12] for some conditions on the term-structures of the parameters (in maturity) which ensure an arbitrage-free surface [13]. These limitations restrict the space of admissible parameters and therefore this solution only solves (4) and partially (1).

1.1.3. Discrete local volatility. One approach to impose the arbitrage-freeness in strike and maturity is to start (again) with a non-homogenous risk-neutral model. One can use a discrete local volatility [1]. Given a time grid of expiries $0:=t_{0}<t_{1}<\cdots<t_{n}$, call prices $c\left(t_{i}, \cdot\right)$ at time $t_{i+1}$ are then taken to be solutions of the ODE:

$$
\left[1-\frac{1}{2} \Delta t_{i} \sigma_{i}(k)^{2} \partial_{k}^{2}\right] c\left(t_{i+1}, k\right)=c\left(t_{i}, k\right), \quad c(0, k)=\left(S_{0}-k\right)_{+} .
$$

By using for $\sigma_{i}(\cdot)$ a piecewise constant function, we can try to match market prices of call options. As pointed in [19], "this method uses a fully implicit finite-difference scheme to compute the probability density of the underlying, stepping forward in time and calibrating model parameters by a least-squares algorithm. Since the size of time step is determined by market quotes, it cannot be reduced arbitrarily, so that, while very instructive, this method clearly has limited accuracy". For example, with this algorithm, we were not able to calibrate equity Vanillas exhibiting a Mexican hat form (see Figure 11), just before earning dates. Some improvements have been considered in [19.

1.1.4. Sinkhorn algorithm. This algorithm [24] has been popularized recently for solving quickly optimal transportation problems by [8]. This algorithm consists in solving an optimal transport problem by including an entropy term in order to make it strictly convex, and then the dual of this entropic optimal transport problem is solved by doing alternatively projections on the marginal distributions of the two measures transported on each other. It has been a quite hot research topic lately, (see for example [22], [21], or [23] for amazing practical approaches). A Sinkhorn's algorithm including the martingale constraint was introduced by [14] in one dimension, and [9] in multi-dimensions with practical approaches. In these works, a third projection on the martingale constraint is introduced and allows to quickly solve the martingale optimal transport problem.

1.2. Contents. In this paper, we will build a solution satisfying (1-2-3-4) by construction. The conditions (1-2-3) are automatically (and exactly) satisfied as we construct a non-parametric density fitting the Vanillas. Our approach is close in spirit to the "Weighted Monte-Carlo approach" based on an entropic penalisation as introduced in [3. However, our approach use a non-degeneracy hypothesis in order to prove the existence of smooth fitting probability. We build the first fitting marginal in Section 3 to help the reader accommodate with the concepts. Then we do the general 
case in Section 4 we assume that the first marginal is created, and we build the second marginal. Furthermore, we present a Sinkhorn's algorithm compatible with the convex order property. The convergence of our algorithm is then proved (see Theorem 4.5) with a fast decay rate and therefore our numerical scheme solves (4). We conclude with numerous examples of fitting to Equity Vanillas for various stocks and indices. Then Section 5 is dedicated to the proofs of the technical results.

\section{Axiomatics: Formulation}

Prices of call options for different maturities $t_{1}<\cdots<t_{n}$ and different strikes are quoted on the market. We denote by $\mathcal{C}_{i}^{K}$ the market prices of maturity $t_{i}$ and strike $K \in \mathcal{K}_{i}$. The set $\mathcal{K}_{i}$ corresponds to the strikes $K_{i}^{1}<\cdots<K_{i}^{n_{i}}$. We denote $\mathcal{P}(\mathcal{X})$ the probability measures on a set $\mathcal{X}$. Building an arbitrage-free implied volatility is equivalent to finding a martingale probability measure $\mathbb{P}^{*} \in \mathcal{P}\left(\mathbb{R}_{+}^{n}\right)$ that matches (exactly) this market prices: $\mathbb{P}^{*}$ should belong to the convex set

$\mathcal{M}_{n}=\left\{\mathbb{P}: \mathbb{E}^{\mathbb{P}}\left[\left(S_{t_{i}}-K\right)_{+}\right]=\mathcal{C}_{i}^{K}, \quad \forall K \in \mathcal{K}_{i}, \mathbb{E}^{\mathbb{P}}\left[S_{t_{i}} \mid S_{0}, \cdots, S_{t_{i-1}}\right]=S_{t_{i-1}}, \quad i=1, \cdots, n\right\}$.

For use below, we set $\mathcal{C}_{i}^{j}:=\mathcal{C}_{i}^{K_{i}^{j}}$ and define the prices of Vertical Spreads (VS), Calendar Vertical Spreads (CVS), and Calendar Butterfly Spreads (CBS):

$$
\begin{aligned}
\mathrm{VS}_{i}^{j} & :=\frac{\mathcal{C}_{i}^{j-1}-\mathcal{C}_{i}^{j}}{K_{i}^{j}-K_{i}^{j-1}} \quad 1 \leq j \leq n_{i}, \\
\mathrm{VS}_{i}^{0} & :=1 \\
\mathrm{CVS}_{i_{1}, i_{2}}^{j_{1}, j_{2}} & :=C_{i_{2}}^{j_{2}}-C_{i_{1}}^{j_{1}}, \\
\mathrm{CBS}_{i, i_{1}, i_{2}}^{j, j_{1}, j_{2}} & :=\frac{C V S_{i_{1}, i}^{j_{1}, j}}{K_{i_{1}}^{j_{1}}-K_{i}^{j}}-\frac{C V S_{i, i_{2}}^{j, j_{2}}}{K_{i}^{j}-K_{i_{2}}^{j_{2}}} .
\end{aligned}
$$

For completeness, we cite the following result that gives necessary and sufficient conditions for arbitrage-freeness:

Lemma 2.1 (see [6, 7] for proofs). $\mathcal{M}_{n}$ is non-empty if and only if for all $i=1, \cdots, n$

(1)

$$
\begin{aligned}
& \mathcal{C}_{i}^{j} \geq 0, \quad 0 \leq j \leq n_{i}, \\
& \operatorname{VS}_{i}^{j} \in[0,1], \quad 1 \leq j \leq n_{i}, \\
& \operatorname{VS}_{i}^{j}>0 \quad \text { if } \quad \forall 1 \leq j \leq n_{i}, \text { we have } C_{i}^{j-1}>0 .
\end{aligned}
$$

(2) $\forall i_{1}, i_{2} \in[1, n]$ s.t. $i_{1}<i_{2}, \forall j_{1} \in\left[0, n_{i_{1}}\right], \forall j_{2} \in\left[0, n_{i_{2}}\right]$

$$
\begin{aligned}
& \mathrm{CVS}_{i_{1}, i_{2}}^{j_{1}, j_{2}} \geq 0, \quad \text { if } \quad K_{i_{1}}^{j_{1}} \geq K_{i_{2}}^{j_{2}}, \\
& \mathrm{CVS}_{i_{1}, i_{2}}^{j_{1}, j_{2}}>0, \quad \text { if } K_{i_{1}}^{j_{1}}>K_{i_{2}}^{j_{2}} \text { and } \mathcal{C}_{i_{1}}^{j_{1}}>0 .
\end{aligned}
$$


(3) $\forall i, i_{1}, i_{2} \in[1, n]$ s.t. $i \leq i_{1}$ and $i \leq i_{2}, \forall j \in\left[0, n_{i}\right], \forall j_{1} \in\left[0, n_{i_{1}}\right], \forall j_{2} \in\left[0, n_{i_{2}}\right]$ s.t. $K_{i_{1}}^{j_{1}}<K_{i}^{j}<K_{i_{2}}^{j_{2}}$ :

$$
\mathrm{CBS}_{i, i_{1}, i_{2}}^{j, j_{1}, j_{2}} \geq 0
$$

Markovian solutions. As a simplification, we could assume that $\mathbb{P}^{*}$ should satisfy a Markov property and therefore belongs instead to the subset of $\mathcal{M}_{n}$ :

$\mathcal{M}_{n}^{\text {Markov }}=\left\{\mathbb{P} \in \mathcal{P}^{\text {Markov }}\left(\mathbb{R}_{+}^{n}\right): \mathbb{E}^{\mathbb{P}}\left[\left(S_{t_{i}}-K\right)_{+}\right]=\mathcal{C}_{i}^{K}, \forall K \in \mathcal{K}_{i}, \mathbb{E}^{\mathbb{P}}\left[S_{t_{i}} \mid S_{t_{i-1}}\right]=S_{t_{i-1}}, i=1, \cdots, n\right\}$.

Where $\mathcal{P}^{\text {Markov }}\left(\mathbb{R}_{+}^{n}\right)$ is the set of probability measures of $\mathcal{P}\left(\mathbb{R}_{+}^{n}\right)$ that satisfy the Markov property.

Lemma 2.2. $\mathcal{M}_{n}^{\text {Markov }}$ is non-empty if and only $\mathcal{M}_{n}$ is non-empty. In particular if the market data $\left(\mathcal{C}_{i}\right)_{1 \leq i \leq n}$ are arbitrage-free, they can be attained by a martingale measure in $\mathcal{M}_{n}^{\text {Markov }}$.

\section{Proof.}

$\Longrightarrow$ : obvious.

$\Longleftarrow$ Take $\mathbb{P} \in \mathcal{M}_{n}$. Then by disintegration, define the marginals $\mathbb{P}^{i-1}$ and $\mathbb{P}^{i}$, which are in the convex order. From Strassen theorem [25], we can build a martingale measure $\mathbb{P}^{i-1, i}$ with marginals $\mathbb{P}^{i-1}$ and $\mathbb{P}^{i}$ (see e.g. [17, or [18] for an explicit construction). By gluing these measures, we get an element in $\mathcal{M}_{n}^{\text {Markov }}$.

2.1. Sequential construction. From the Markov property, an element $\mathbb{P} \in \mathcal{M}_{n}^{\text {Markov }}$ could be written as

$$
\mathbb{P}\left(d s_{1}, \cdots, d s_{n}\right)=\mathbb{P}^{1}\left(d s_{1}\right) \prod_{i=2}^{n} \mathbb{P}^{i-1, i}\left(d s_{i} \mid d s_{i-1}\right),
$$

where the probability $\mathbb{P}^{1}$ and $\left(\mathbb{P}^{i-1, i}\right)_{i=1, \cdots, n}$ are constructed as follows:

(1) We choose a $\mathbb{P}^{1} \in \mathcal{M}_{1}^{\text {Markov }}$ (a specific example is constructed in Section 3 ) with

$$
\mathcal{M}_{1}^{\text {Markov }}:=\left\{\mathbb{P} \in \mathcal{P}\left(\left\{S_{0}\right\} \times \mathbb{R}_{+}\right) \quad: \quad \mathbb{E}^{\mathbb{P}}\left[\left(S_{t_{1}}-K\right)_{+}\right]=\mathcal{C}_{1}^{K}, \quad \forall K \in \mathcal{K}_{1}, \quad \mathbb{E}^{\mathbb{P}}\left[S_{t_{1}} \mid S_{0}\right]=S_{0}\right\} .
$$

(2) We choose a $\mathbb{P}^{1,2} \in \mathcal{M}_{1,2}^{\text {Markov }}$ (a specific example is constructed in Section 4) with

$$
\mathcal{M}_{1,2}^{\text {Markov }}\left(\mathbb{P}^{1}\right):=\left\{\mathbb{P} \in \mathcal{P}\left(\mathbb{R}_{+}^{2}\right): S_{t_{1}} \stackrel{\mathbb{P}}{\sim} \mathbb{P}^{1}, \quad \mathbb{E}^{\mathbb{P}}\left[\left(S_{t_{2}}-K\right)_{+}\right]=\mathcal{C}_{2}^{K}, \quad \mathbb{E}^{\mathbb{P}}\left[S_{t_{2}} \mid S_{t_{1}}\right]=S_{t_{1}}\right\} .
$$

From $\mathbb{P}^{1,2} \in \mathcal{M}_{1,2}^{\text {Markov }}$, we define $\mathbb{P}^{2}$ as

$$
\mathbb{P}^{2}\left(d s_{2}\right)=\int \mathbb{P}^{1,2}\left(d s_{1}, d s_{2}\right) .
$$

(3) We iterate step (2) to obtain $\left(\mathbb{P}^{i-1, i}\right)_{i=3, \cdots, n}$.

Remark 2.3. Notice that the probability measures in $\mathcal{M}_{1}^{\text {Markov }}$ and $\mathcal{M}_{1,2}^{\text {Markov }}\left(\mathbb{P}^{1}\right)$ are trivially Markov, as they are on two times. 
2.2. Adding bid-ask prices. In practice, market prices are quoted with bid-ask prices. Our discussion can be generalized to this case by replacing $\mathcal{M}_{1}^{\text {Markov }}$ and $\mathcal{M}_{1,2}^{\text {Markov }}$ by

$\widetilde{\mathcal{M}}_{1}^{\text {Markov }}=\left\{\mathbb{P} \in \mathcal{P}\left(\left\{S_{0}\right\} \times \mathbb{R}_{+}\right): \mathcal{C}_{1}^{K, \text { bid }} \leq \mathbb{E}^{\mathbb{P}}\left[\left(S_{t_{1}}-K\right)_{+}\right] \leq \mathcal{C}_{1}^{K, \text { ask }}, \forall K \in \mathcal{K}_{1}, \mathbb{E}^{\mathbb{P}}\left[S_{t_{1}} \mid S_{0}\right]=S_{0}\right\}$.

$\widetilde{\mathcal{M}}_{1,2}^{\text {Markov }}\left(\mathbb{P}^{1}\right)=\left\{\mathbb{P}: S_{t_{1}} \stackrel{\mathbb{P}}{\sim} \mathbb{P}^{1}, \mathbb{E}^{\mathbb{P}}\left[S_{t_{2}} \mid S_{t_{1}}\right]=S_{t_{1}}, \mathcal{C}_{2}^{K, \text { bid }} \leq \mathbb{E}^{\mathbb{P}}\left[\left(S_{t_{2}}-K\right)_{+}\right] \leq \mathcal{C}_{2}^{K, \text { ask }}, \forall K \in \mathcal{K}_{2}\right\}$.

We consider this setup in the next sections. The arbitrage-free conditions, which ensure that $\widetilde{\mathcal{M}}_{1,2}^{\text {Markov }}\left(\mathbb{P}^{1}\right)$ is non-empty, are given in $[7$ : we can take the same conditions than in Lemma 2.1 with redefining

$$
\begin{aligned}
\mathrm{VS}_{i}^{j} & :=\frac{\mathcal{C}_{i}^{K_{j-1}, \text { bid }}-\mathcal{C}_{i}^{K_{j}, \text { ask }}}{K_{i}^{j}-K_{i}^{j-1}} 1 \leq j \leq n_{i}, \\
\mathrm{CVS}_{i_{1}, i_{2}}^{j_{1}, j_{2}} & :=C_{i_{2}}^{K_{j_{2}}, \text { ask }}-C_{i_{1}}^{K_{j_{1}}, \text { bid }}, \\
\mathrm{CBS}_{i, i_{1}, i_{2}}^{j, j_{1}, j_{2}} & :=\frac{C_{i}^{K_{j}, \text { ask }}-C_{i_{1}}^{K_{j_{1}}, \text { bid }}}{K_{i_{1}}^{j_{1}}-K_{i}^{j}}-\frac{C_{i_{2}}^{K_{j_{2}}, \text { bid }}-C_{i}^{K_{j}, \text { ask }}}{K_{i}^{j}-K_{i_{2}}^{j_{2}}} .
\end{aligned}
$$

Remark 2.4 (Cash/yield dividends). We assume here that the spot process $S_{t}$ jumps down by the dividend amounts $D_{i}\left(S_{\tau_{i}^{-}}\right)=\beta_{i} S_{\tau_{i}^{-}}+\alpha_{i}$, paid at the dates $0<\tau_{1}<\ldots \tau_{n}<T$, and that between dividend dates it follows a diffusion. By setting $S_{t}=A(t)+B(t) X_{t}$ (see [16] for formulas for $A$ and $B$ as functions of $\left(\alpha_{i}, \beta_{i}\right)$ ), one obtains that $X_{t}$ is a martingale. Call options on $S$ can therefore be written as call options on $X$. One can then applies our construction to $X$ and deduce then call options on $S$. Using this mapping, we will assume no dividends/zero rates in the following.

\section{Building AN Element in $\widetilde{\mathcal{M}}_{1}^{\text {Markov }}$}

For the sake of simplicity for the rest of this paper, we denote $S_{1}:=S_{t_{1}}$ and $S_{2}:=S_{t_{2}}$. An element $\mathbb{P}^{*} \in \widetilde{\mathcal{M}}_{1}^{\text {Markov }}$ can be obtained by minimizing a convex lower semi-continuous functional $\mathcal{F}_{1}$ :

$$
\inf _{\mathbb{P} \in \widetilde{\mathcal{M}}_{1}^{\text {Markov }}} \mathcal{F}_{1}(\mathbb{P})=\mathcal{F}_{1}\left(\mathbb{P}^{*}\right), \quad \mathbb{P}^{*} \in \widetilde{\mathcal{M}}_{1}^{\text {Markov }} .
$$

3.1. Choice of $\mathcal{F}_{1}$. We choose $\left(\omega_{K}\right)_{K \in \mathcal{K}_{1}} \in \mathbb{R}_{+}^{n_{1}}$ and consider the regularized Kullback-Leibler functional:

$$
\mathcal{F}_{1}(\mathbb{P}):=\mathbb{E}^{\mathbb{P}}\left[\ln \frac{d \mathbb{P}}{d m_{0}}-1\right]+\sum_{K \in \mathcal{K}_{1}} \frac{1}{2 \omega_{K}}\left(\mathbb{E}^{\mathbb{P}}\left[\left(S_{1}-K\right)_{+}\right]-\mathcal{C}_{1}^{K, \text { mid }}\right)^{2},
$$

where we denote $\mathcal{C}_{1}^{K \text {,mid }}:=\frac{\mathcal{C}_{1}^{K, \text { bid }}+\mathcal{C}_{1}^{K \text {,ask }}}{2}$. This equation depends on a prior measure $m_{0}$ on $\mathbb{R}_{+}$, left unspecified for the moment. Notice that by introducing dual variables $v_{K} \in \mathbb{R}$, for each $K \in \mathcal{K}_{1}$, therefore $\mathcal{F}_{1}$ may also be written as

$$
\mathcal{F}_{1}(\mathbb{P}):=\mathbb{E}^{\mathbb{P}}\left[\ln \frac{d \mathbb{P}}{d m_{0}}-1\right]-\inf _{v \in \mathbb{R}^{\mathcal{K}_{1}}} \sum_{K \in \mathcal{K}_{1}} v_{K}\left(\mathcal{C}_{1}^{K, \text { mid }}-\mathbb{E}^{\mathbb{P}}\left[\left(S_{1}-K\right)_{+}\right]\right)+\frac{1}{2} v_{K}^{2} \omega_{K} .
$$

Notice that we choose the form $\mathbb{E}^{\mathbb{P}}\left[\ln \frac{d \mathbb{P}}{d m_{0}}-1\right]$ over $\mathbb{E}^{\mathbb{P}}\left[\ln \frac{d \mathbb{P}}{d m_{0}}\right]$ because it gives exactly the same solutions, but with simpler formulas. 
The following condition will guarantee the existence of solutions for 3.2 Let $0<K_{1}<\ldots<K_{k}$, $\mathcal{K}:=\left\{K_{i}: 1 \leq i \leq k\right\}$, and $\mathbb{P}$ a probability distribution on $\mathbb{R}$ with finite support $\mathcal{X}$.

Definition 3.1. We say that $\left(m_{0}, \mathbb{P},\left(\mathcal{C}^{K \text {,bid/ask }}\right)_{K \in \mathcal{K}}\right)$ is non-degenerate if up to denoting $K_{0}:=0$ and $K_{k+1}:=\infty$, and setting $\mathcal{C}^{0, \text { bid }}:=\mathcal{C}^{0 \text {,ask }}:=\mathbb{E}^{\mathbb{P}}\left[S_{1}\right]$ and $\mathcal{C}^{\infty, \text { bid }}:=\mathcal{C}^{\infty \text {,ask }}:=1$, we may find $\mathcal{C} \in \mathbb{R}^{k+2}$ such that for all $0 \leq i \leq k+1$, we have

(i) $\mathcal{C}^{K_{i}, \text { bid }} \leq \mathcal{C}_{i} \leq \mathcal{C}^{K_{i}, \text { ask }}$,

(ii) $\left(M_{\text {call }}^{-1} \mathcal{C}\right)_{i}>0$,

(iii) $\mathcal{C}_{i}>\mathbb{E}^{\mathbb{P}^{1}}\left[\left(S_{1}-K_{i}\right)_{+}\right]$, if $1 \leq i \leq k$,

(iv) the projection of $m_{0}$ on $S_{1}$ has support $\mathcal{X}$,

(v) $m_{0}\left[\{s\} \times\left(K_{i}, K_{i+1}\right)\right]>0$, for $s \in \mathcal{X}$ if $i \leq k$,

(vi) $m_{0}[\{s\} \times(-\infty, s)]>0$ and $m_{0}[\{s\} \times(s, \infty)]>0$, for $s \in \mathcal{X}$,

where $M_{\text {call }}:=\left(\left(K_{j}-K_{i}\right)_{+}\right)_{0 \leq i, j \leq k+1}$, with the convention $\left(K_{k+1}-K_{i}\right)_{+}:=\left(K_{i}-K_{k+1}\right)_{+}:=1$ for all $i$.

Remark 3.2. Notice that (ii) in Definition 3.1 is equivalent to a kind of no-arbitrage condition: it is equivalent to the fact that if we define a non-negative payoff $f(s):=\sum_{i=0}^{k+1} \lambda_{i}\left(s-K_{i}\right)_{+} \geq 0$, then we have $\sum_{i=0}^{k+1} \lambda_{i} \mathcal{C}_{i} \geq 0$, with equality if and only if $\lambda_{0}=\ldots=\lambda_{k+1}=0$.

Proposition 3.3. We assume that $\left(m_{0}, \delta_{S_{0}},\left(\mathcal{C}_{1}^{K \text {,bid/ask }}\right)_{K \in \mathcal{K}_{1}}\right)$ is non-degenerate.

Then the minimisation 3.2) is attained by $\mathbb{P}^{*} \in \widetilde{\mathcal{M}}_{1}^{\text {Markov }}$ with

$$
\mathbb{P}^{*}(d s)=m_{0}(d s) e^{-\sum_{K \in \mathcal{K}_{1}} V_{K}^{*}(s-K)_{+}-u_{0}^{*}-h_{0}^{*}\left(s-S_{0}\right)},
$$

where $\left(V_{K}^{*}\right)_{K \in \mathcal{K}_{1}}, u_{0}^{*}$, and $h_{0}^{*}$ solve the strictly convex unconstrained minimization:

$$
\begin{aligned}
& \inf _{V \in \mathbb{R}^{\mathcal{K}_{1}, u_{0}, h_{0} \in \mathbb{R}}} \mathcal{G}_{1}\left(u_{0}, h_{0}, V\right), \\
& \text { where } \mathcal{G}_{1}\left(u_{0}, h_{0}, V\right):=u_{0}+\sum_{K \in \mathcal{K}_{1}} f_{1}^{K, \text { bid } / \text { ask }}\left(V_{K}, \omega_{K}\right)+\sum_{K \in \mathcal{K}_{1}} V_{K} \mathcal{C}_{1}^{K, \text { mid }} \\
& +\mathbb{E}^{m_{0}}\left[e^{-\sum_{K \in \mathcal{K}_{1}} V_{K}\left(S_{1}-K\right)_{+}-u_{0}-h_{0}\left(S_{1}-S_{0}\right)}\right] \text {, } \\
& \text { and } f_{1}^{K, \text { bid/ask }}(V, \omega):=\quad \frac{V^{2} \omega}{2}, \quad \text { if } \Delta \mathcal{C}_{1}^{K, \text { bid }} \leq V \omega \leq \Delta \mathcal{C}_{1}^{K \text {,ask }} \\
& :=\Delta \mathcal{C}_{1}^{K, \text { ask }} V-\frac{\left(\Delta \mathcal{C}_{1}^{K, \text { ask }}\right)^{2}}{2 \omega}, \text { if } \Delta \mathcal{C}_{1}^{K, \text { ask }}<V \omega \\
& :=\Delta \mathcal{C}_{1}^{K, \text { bid }} V-\frac{\left(\Delta \mathcal{C}_{1}^{K, \text { bid }}\right)^{2}}{2 \omega}, \text { if } \Delta \mathcal{C}_{1}^{K, \text { bid }}>V \omega .
\end{aligned}
$$

Here $\Delta \mathcal{C}_{1}^{\text {bid/ask }}:=\mathcal{C}_{1}^{\text {bid/ask }}-\mathcal{C}_{1}^{\text {mid }}$.

The proof of Proposition 3.3 is reported to Section 5. 
Dependence on the prior. We consider two prior densities $\mathbb{P}_{0}$ and $\mathbb{P}_{0}^{\prime}$. By definition, the vanillas constructed using the two priors satisfy the equations for all $K \in \mathcal{K}_{1}$ :

$$
\begin{aligned}
& \mathcal{C}_{1}^{K, \text { mid }}+\partial_{V} f\left(V_{K}, \omega_{K}\right)-\mathcal{C}^{\text {model }}\left(K, \mathbb{P}_{0}\right)=0 \\
& \mathcal{C}_{1}^{K, \text { mid }}+\partial_{V} f\left(V_{K}^{\prime}, \omega_{K}\right)-\mathcal{C}^{\text {model }}\left(K, \mathbb{P}_{0}^{\prime}\right)=0
\end{aligned}
$$

By taking the difference, we get

$$
\begin{aligned}
\left|\mathcal{C}^{\text {model }}\left(K, \mathbb{P}_{0}^{\prime}\right)-\mathcal{C}^{\text {model }}\left(K, \mathbb{P}_{0}\right)\right| & =\left|\partial_{V} f\left(V_{K}^{\prime}, \omega_{K}\right)-\partial_{V} f\left(V_{K}, \omega_{K}\right)\right| \\
& \leq \omega_{K}\left|V_{K}^{\prime}-V_{K}\right|
\end{aligned}
$$

3.2. Numerical examples. In practice, we take $\omega_{K}=\Lambda\left|\mathcal{C}_{1}^{K, \text { ask }}-\mathcal{C}_{1}^{K, \text { bid }}\right|$ with $\Lambda=0.1$ in our numerical examples. The minimization over $V$ is performed using a modified Newton method and a user-supplied Hessian. In order to have easier computations thanks to the closed formulas displayed in Remark 4.3 , we use as a reference measure $m_{0}\left(d s_{1}\right):=\mathbb{P}_{0}\left(d s_{1}\right) \mathbf{1}_{s_{1} \geq 0}$, where $\mathbb{P}_{0}$ is the Gaussian measure $\mathcal{N}\left(S_{0}, \sigma_{0}^{2} t_{1}\right)$, properly normalized on $\mathbb{R}_{+}$, and where $\sigma_{0}$ is chosen to minimize the criterion:

$$
\inf _{\sigma_{0}} \sum_{K \in \mathcal{K}_{1}}\left(\mathbb{E}^{\mathbb{P}_{0}}\left[\left(S_{1}-K\right)_{+}\right]-\mathcal{C}_{1}^{K, \text { mid }}\right)^{2}=\inf _{\sigma_{0}} \sum_{K \in \mathcal{K}_{1}}\left(\mathrm{~B}\left(S_{0}, t_{1}, K, \sigma_{0}\right)-\mathcal{C}_{1}^{K, \mathrm{mid}}\right)^{2},
$$

with

$$
\mathrm{B}(s, t, K, \sigma):=\frac{1}{2}(s-K) \operatorname{erf}\left(\frac{K-s}{\sqrt{2} \sigma \sqrt{t}}\right)+\frac{\sigma \sqrt{t} e^{-\frac{(K-s)^{2}}{2 \sigma^{2} t}}}{\sqrt{2 \pi}} .
$$

In Figure 1, we show examples of calibration with two stocks (Google \& Amazon) near earnings. By construction, the fit is perfect (within the bid/ask spread) and arbitrage-free. In Figure 3.2 . we consider two indices (Dax \& Euro Stoxx 50).
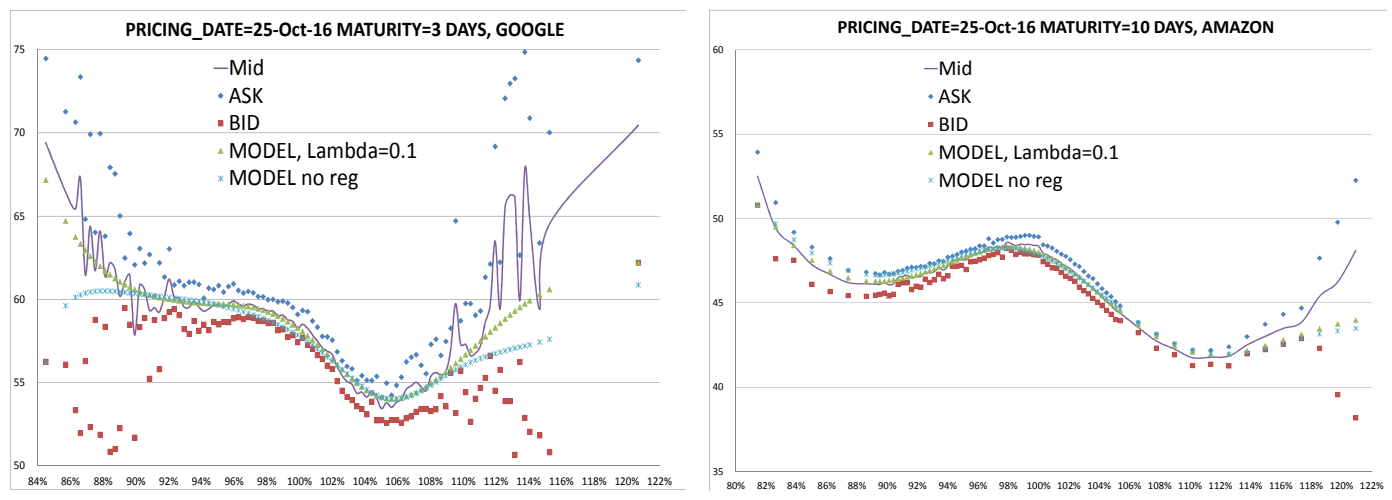

Figure 1. Computational time $=0.1 \mathrm{~s}$. Left: GOOGLE. Right: AMAZON. The plots denoted "Model no reg" mean that we have chosen $\Lambda=\infty$. 

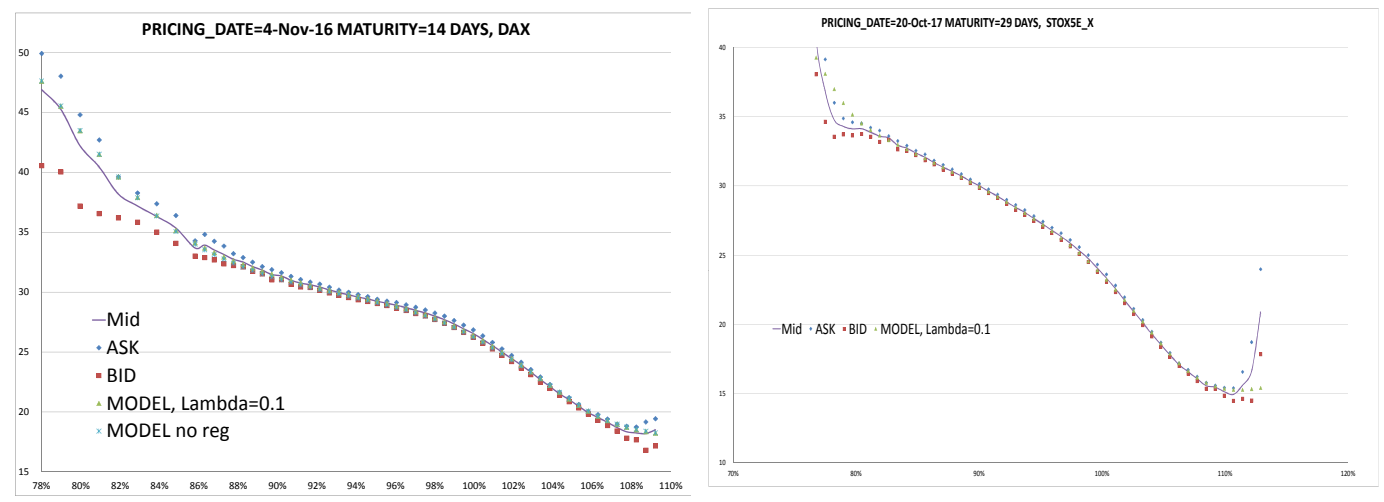

Figure 2. Computational time $=0.1 \mathrm{~s}$. Left: DAX. Right: EURO STOXX 50.

\section{Building an element in $\widetilde{\mathcal{M}}_{12}^{\text {Markov }}\left(\mathbb{P}^{1}\right)$}

Proceeding as in the previous section, we consider the minimization problem:

$$
\inf _{\mathbb{P} \in \widetilde{\mathcal{M}}_{12}^{\text {Markov }}\left(\mathbb{P}^{1}\right)} \mathbb{E}^{\mathbb{P}}\left[\ln \frac{d \mathbb{P}}{d m_{0}}-1\right]+\sum_{K \in \mathcal{K}_{2}} \frac{1}{2 \omega_{K}}\left(\mathbb{E}^{\mathbb{P}}\left[\left(S_{2}-K\right)_{+}\right]-\mathcal{C}_{2}^{K, \text { mid }}\right)^{2},
$$

depending on a prior measure $m_{0}$ on $\left(\mathbb{R}_{+}\right)^{2}$, left unspecified for the moment. Similar to $f_{1}^{K \text {,bid/ask }}$, we define

$$
\begin{aligned}
& f_{2}^{K, \text { bid/ask }}(V, \omega):=\quad \frac{V^{2} \omega}{2}, \quad \text { if } \Delta \mathcal{C}_{2}^{K, \text { bid }} \leq V \omega \leq \Delta \mathcal{C}_{2}^{K, \text { ask }} \\
& :=\Delta \mathcal{C}_{2}^{K, \text { ask }} V-\frac{\left(\Delta \mathcal{C}_{2}^{K, \text { ask }}\right)^{2}}{2 \omega}, \quad \text { if } \Delta \mathcal{C}_{2}^{K, \text { ask }}<V \omega \\
& :=\Delta \mathcal{C}_{2}^{K, \text { bid }} V-\frac{\left(\Delta \mathcal{C}_{2}^{K, \text { bid }}\right)^{2}}{2 \omega}, \text { if } \Delta \mathcal{C}_{2}^{K, \text { bid }}>V \omega .
\end{aligned}
$$

Similar to Proposition 3.3 we obtain

Proposition 4.1. We assume that $\left(m_{0}, \mathbb{P}^{1},\left(\mathcal{C}_{2}^{K \text {,bid/ask }}\right)_{K \in \mathcal{K}_{2}}\right)$ is non-degenerate.

Then the minimization 4.3 is attained by $\mathbb{P}^{*} \in \widetilde{\mathcal{M}}_{12}^{\text {Markov }}\left(\mathbb{P}^{1}\right)$ with

$$
\mathbb{P}^{*}\left(d s_{1}, d s_{2}\right)=m_{0}\left(d s_{1}, d s_{2}\right) e^{-\sum_{K \in \mathcal{K}_{2}} V_{K}^{*}\left(s_{2}-K\right)_{+}-u_{1}^{*}\left(s_{1}\right)-h_{1}^{*}\left(s_{1}\right)\left(s_{2}-s_{1}\right)},
$$

where $u_{1}^{*}, h_{1}^{*}$, and $V^{*}$ solve the strictly convex unconstrained minimization:

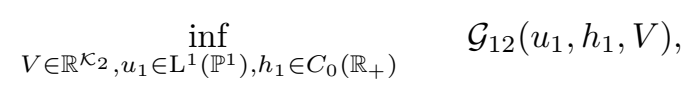

where

$$
\begin{aligned}
\mathcal{G}_{12}\left(u_{1}, h_{1}, V\right):= & \mathbb{E}^{\mathbb{P}^{1}}\left[u_{1}\left(S_{1}\right)\right]+\sum_{K \in \mathcal{K}_{2}} f_{2}^{K, \text { bid } / \text { ask }}\left(V_{K}, \omega_{K}\right)+\sum_{K \in \mathcal{K}_{2}} V_{K} \mathcal{C}_{2}^{K, \text { mid }} \\
& +\mathbb{E}^{m_{0}}\left[e^{-\sum_{K \in \mathcal{K}_{2}} V_{K}\left(S_{2}-K\right)_{+}-u_{1}\left(S_{1}\right)-h\left(S_{1}\right)\left(S_{2}-S_{1}\right)}\right] .
\end{aligned}
$$

The proof of Proposition 4.1 is reported to Section 5 . 
Remark 4.2. Notice that Proposition 3.3 can be seen as a particular case of Proposition 4.1 with $\mathbb{P}_{1}$ Dirac measure in $S_{0}$.

To solve this problem in practice, we assume up to approximate $\mathbb{P}^{1}$ and the marginal distribution of $m_{0}$ on $S_{1}$, that they have a finite support $\mathcal{S}_{1} \subset \mathbb{R}_{+}$. Let $s_{1} \in \mathcal{S}_{1}$, the zero of the gradient with respect to $u_{1}$ is given by the equation:

$$
e^{-u_{1}\left(s_{1}\right)}=\frac{\mathbb{P}^{1}\left(s_{1}\right)}{I_{u}\left(h\left(s_{1}\right), V(\cdot), s_{1}\right)}
$$

where we have set

$$
I_{u}\left(\theta, V, s_{1}\right):=\int e^{-\sum_{K \in \mathcal{K}_{2}} V_{K}\left(s_{2}-K\right)_{+}-\theta\left(s_{2}-s_{1}\right)} m_{0}\left(s_{1}, d s_{2}\right)=e^{u_{1}\left(s_{1}\right)} \partial_{u_{1}\left(s_{1}\right)} \mathcal{G}_{12}\left(u_{1}, h_{1}, V\right),
$$

The zero of the gradient with respect to $h\left(s_{1}\right)$ is given by the equation: $h\left(s_{1}\right):=\theta$ is the unique zero of

$$
I_{h}\left(\theta, V, s_{1}\right):=0,
$$

where

$I_{h}\left(\theta, V, s_{1}\right):=\int e^{-\sum_{K \in \mathcal{K}_{2}} V_{K}\left(s_{2}-K\right)_{+}-\theta\left(s_{2}-s_{1}\right)}\left(s_{2}-s_{1}\right) m_{0}\left(s_{1}, d s_{2}\right)=e^{u_{1}\left(s_{1}\right)} \partial_{h_{1}\left(s_{1}\right)} \mathcal{G}_{12}\left(u_{1}, h_{1}, V\right)$.

In practice, this may be done thanks to a 1D Newton algorithm on the function $h_{1}\left(s_{1}\right) \mapsto$ $\min _{h\left(s_{1}\right)} \mathcal{G}_{12}\left(u_{1}, h_{1}, V\right)$, see Subsections 3.3.3 and 3.3.5 in [9].

For use below, we also introduce for all $Q \in \mathcal{K}_{2}$ :

$I_{Q}\left(h_{1}, V, s_{1}\right):=\int\left(s_{2}-Q\right)_{+} e^{-\sum_{K \in \mathcal{K}_{2}} V_{K}\left(s_{2}-K\right)_{+}-h_{1}\left(s_{2}-s_{1}\right)} m_{0}\left(s_{1}, d s_{2}\right)=e^{u_{1}\left(s_{1}\right)} \partial_{V_{Q}} \mathcal{G}_{12}\left(u_{1}, h_{1}, V\right)$.

4.1. Speed-up: Choice of a prior. We take $m_{0}\left(d s_{1}, d s_{2}\right)=\mathbf{1}_{s_{1} \geq 0} \mathbb{P}_{\sigma_{0}}\left(d s_{1}\right) \mathbb{P}\left(d s_{2} \mid s_{1}\right)$ where $\mathbb{P}_{\sigma_{0}}\left(d s_{1}\right)$ is a normal density with volatility $\sigma_{0}$ and under $\mathbb{P}$ :

$$
S_{2}=S_{1}+\sigma\left(S_{1}\right) \sqrt{t_{2}-t_{1}} Z, \quad Z \in \mathrm{N}(0,1), \quad \sigma\left(S_{1}\right)=\sigma_{0} S_{1}^{\beta},
$$

where $\sigma_{0}$ and $\beta$ are two parameters. We choose $\sigma_{0}$ and $\beta$ by minimizing the least-square problem:

$$
\begin{aligned}
& \inf _{\sigma_{0}, \beta} \sum_{K \in \mathcal{K}_{2}}\left(\mathbb{E}^{m_{0}}\left[\left(S_{2}-K\right)_{+}\right]-\mathcal{C}_{2}^{K, \text { mid }}\right)^{2} \\
= & \inf _{\sigma_{0}, \beta} \sum_{K \in \mathcal{K}_{2}}\left(\mathbb{E}^{\mathbb{P}_{\sigma_{0}}}\left[\mathrm{~B}\left(S_{1}, t_{2}-t_{1}, K, \sigma_{0} S_{1}^{\beta}\right)\right]-\mathcal{C}_{2}^{K, \text { mid }}\right)^{2},
\end{aligned}
$$

with

$$
\mathrm{B}(s, t, K, \sigma):=\frac{1}{2}(s-K) \operatorname{erf}\left(\frac{K-s}{\sqrt{2} \sigma \sqrt{t}}\right)+\frac{\sigma \sqrt{t} e^{-\frac{(K-s)^{2}}{2 \sigma^{2} t}}}{\sqrt{2 \pi}} .
$$

Notice that by the fact that $S_{2}$ is normally-distributed when conditioned on $S_{1}$, the integration over $s_{2}$ can be performed exactly thanks to the definition of the functions $I_{u}, I_{h}$ and $I_{Q}$, defined above. These functions' values can be written in closed-form. 
Remark 4.3 (Explicit formulas). For completeness, we give the formulas, obtained with Mathematica, that we use in our numerical implementation. Let $A:=\frac{K_{1}-s_{1}}{\sigma}, B:=\frac{K_{2}-s_{1}}{\sigma}$ and $\sigma:=\sigma\left(s_{1}\right) \sqrt{t_{2}-t_{1}}$. We have

$$
\begin{aligned}
& \int_{K_{1}}^{K_{2}} e^{\alpha s_{2}} \mathbb{P}\left(d s_{2} \mid s_{1}\right)=\frac{1}{2} e^{\frac{\alpha^{2} \sigma^{2}}{2}+\alpha s_{1}}\left(\operatorname{erf}\left(\frac{B-\alpha \sigma}{\sqrt{2}}\right)-\operatorname{erf}\left(\frac{A-\alpha \sigma}{\sqrt{2}}\right)\right) . \\
& 2 \sqrt{2 \pi} \int_{K_{1}}^{K_{2}} e^{\alpha s_{2}}\left(s_{2}-s_{1}\right) \mathbb{P}\left(d s_{2} \mid s_{1}\right)=\sigma e^{\alpha s_{1}}\left(2 e^{A \alpha \sigma-\frac{A^{2}}{2}}-\sqrt{2 \pi} \alpha \sigma e^{\frac{\alpha^{2} \sigma^{2}}{2}} \operatorname{erf}\left(\frac{A-\alpha \sigma}{\sqrt{2}}\right)\right. \\
& \left.+\sqrt{2 \pi} \alpha \sigma e^{\frac{\alpha^{2} \sigma^{2}}{2}} \operatorname{erf}\left(\frac{B-\alpha \sigma}{\sqrt{2}}\right)-2 e^{\alpha B \sigma-\frac{B^{2}}{2}}\right) . \\
& 2 \sqrt{2 \pi} \int_{K_{1}}^{K_{2}} e^{\alpha s_{2}}\left(s_{2}-K\right) \mathbb{P}\left(d s_{2} \mid s_{1}\right)=e^{\alpha s_{1}}\left(2 \sigma e^{A \alpha \sigma-\frac{A^{2}}{2}}-\sqrt{2 \pi} e^{\frac{\alpha^{2} \sigma^{2}}{2}} \operatorname{erf}\left(\frac{A-\alpha \sigma}{\sqrt{2}}\right)\left(\alpha \sigma^{2}-K+s_{1}\right)\right. \\
& \left.+\sqrt{2 \pi} e^{\frac{\alpha^{2} \sigma^{2}}{2}} \operatorname{erf}\left(\frac{B-\alpha \sigma}{\sqrt{2}}\right)\left(\alpha \sigma^{2}-K+s_{1}\right)-2 \sigma e^{\alpha B \sigma-\frac{B^{2}}{2}}\right) \cdot \\
& 2 \sqrt{2 \pi} \int_{K_{1}}^{K_{2}} e^{\alpha\left(s_{2}-s_{1}\right)}\left(s_{2}-K\right)\left(s_{2}-Q\right) \mathbb{P}\left(d s_{2} \mid s_{1}\right)=2 \sigma\left(e^{\alpha A \sigma-\frac{A^{2}}{2}}\left(\sigma(\alpha \sigma+A)-K-Q+2 s_{1}\right)\right. \\
& \left.+e^{-\frac{1}{2} B(B-2 \alpha \sigma)}\left(-\sigma(\alpha \sigma+B)+K+Q-2 s_{1}\right)\right) \\
& +\sqrt{2 \pi} e^{\frac{\alpha^{2} \sigma^{2}}{2}}\left(\operatorname{erf}\left(\frac{B-\alpha \sigma}{\sqrt{2}}\right)-\operatorname{erf}\left(\frac{A-\alpha \sigma}{\sqrt{2}}\right)\right)\left(\sigma^{2}-\left(-\alpha \sigma^{2}+K-s_{1}\right)\left(\alpha \sigma^{2}-Q+s_{1}\right)\right) .
\end{aligned}
$$

The last formula is used for computing the hessian $\partial_{V}^{2} \mathcal{G}_{12}$.

Remark 4.4 (Other formulas). Note that we have

$$
\mathbb{E}^{m_{0}}\left[e^{-\sum_{K \in \mathcal{K}_{2}} V_{K}\left(S_{2}-K\right)_{+}-h\left(S_{1}\right)\left(S_{2}-S_{1}\right)-u_{1}\left(S_{1}\right)}\right]=\mathbb{E}^{\mathbb{P} \sigma_{0}}\left[I_{u}\left(h\left(S_{1}\right), V(\cdot), S_{1}\right) e^{-u_{1}\left(S_{1}\right)}\right] .
$$

and

$$
\mathbb{E}^{\mathbb{P}_{\sigma_{0}}}\left[\left(S_{2}-Q\right)_{+} e^{-\sum_{K \in \mathcal{K}_{2}} V_{K}\left(S_{2}-K\right)_{+}-h\left(S_{1}\right)\left(S_{2}-S_{1}\right)-u_{1}\left(S_{1}\right)}\right]=\mathbb{E}^{\mathbb{P} \sigma_{0}}\left[I_{Q}\left(h\left(S_{1}\right), V(\cdot), S_{1}\right) e^{-u_{1}\left(S_{1}\right)}\right] .
$$

4.2. Sinkhorn's algorithm in a nutshell. In order to be able to apply the next algorithm, we need to be able to do computations for each $s_{1}$. In order to do it in practice, we need to introduce an approximation $\mathbb{P}_{\mathcal{X}_{1}}^{1} \approx \mathbb{P}^{1}$ that is supported on a finite grid $\mathcal{X}_{1} \subset \mathbb{R}_{+}$. As our goal is to estimate call prices, that are naturally 1 -Lipschitz, this approximation should be made in terms of Wasserstein distance $\mathcal{W}_{1}$. To make the computation possible in practice, we approximate $\mathbb{P}^{1}$ by $\mathbb{P}_{\mathcal{X}_{1}}^{1}:=\frac{1}{Z} \sum_{s_{1} \in \mathcal{X}_{1}} \frac{d \mathbb{P}^{1}}{d x}\left(s_{1}\right) \mathbf{1}_{s_{1}}$, where $Z:=\sum_{s_{1} \in \mathcal{X}_{1}} \frac{d \mathbb{P}^{1}}{d x}\left(s_{1}\right), \mathcal{X}_{1}:=\left\{a+\frac{k}{n-1}(b-a): 0 \leq k \leq n-1\right\}$, and $\frac{d \mathbb{P}^{1}}{d x}$ denotes the density of $\mathbb{P}^{1}$ (well-defined as $\mathbb{P}^{1}$ is dominated by a normal measure, see 4.1 , where $a<b$ and $n \geq 2$ are carefully chosen parameters in order to make the approximation good enough. If we chose $a$ smaller to the lowest strike $K_{1}^{\min }:=\min \mathcal{K}_{1}$, the error in terms of call prices estimates will therefore be controlled by $\frac{b-a}{n}+\int_{b}^{\infty}\left(x-K_{1}^{\min }\right)_{+} d \mathbb{P}^{1}(x)$, which allows to chose $a$, $b$, and $n$. We do the same approximation for $\mathbb{P}^{\sigma_{0}}$. This one matters less as the projection of this measure on $S_{1}$ will be exactly $\mathbb{P}_{\mathcal{X}_{1}}^{1}$.

I Start with $h_{1}:=0, u_{1}:=0$ and $V_{K}:=0$ for all $K \in \mathcal{K}_{2}$. 
II Projection on $\left(u_{1}, h_{1}\right)$ : Solve equations (4.4) and (4.5) for all $s_{1} \in \mathcal{X}_{1}$.

III Solve the strictly convex smooth finite-dimensional unconstrained minimization over $V$ :

$$
\inf _{V_{K} \in \mathbb{R}} \mathcal{G}_{12}\left(u_{1}, h, V\right),
$$

with

$$
\begin{aligned}
\mathcal{G}_{12}\left(u_{1}, h, V\right):= & \mathbb{E}^{\mathbb{P}_{\mathcal{X}_{1}}^{1}}\left[u_{1}\right]+\sum_{K \in \mathcal{K}_{2}} f_{2}^{K, \text { bid } / \text { ask }}\left(V_{K}, \omega_{K}\right)+\sum_{K \in \mathcal{K}_{2}} V_{K} \mathcal{C}_{2}^{K, \text { mid }} \\
& +\mathbb{E}^{m_{0}}\left[e^{-\sum_{K \in \mathcal{K}_{2}} V_{K}\left(S_{2}-K\right)_{+}-h\left(S_{1}\right)\left(S_{2}-S_{1}\right)-u_{1}\left(S_{1}\right)}\right] .
\end{aligned}
$$

From Remark 4.4 , this can be written exactly as

$$
\begin{aligned}
\mathcal{G}_{12}\left(u_{1}, h_{1}, V\right)= & \mathbb{E}^{\mathbb{P}^{1}} x_{1}\left[u_{1}\right]+\sum_{K \in \mathcal{K}_{2}} f^{K, \text { bid } / \text { ask }}\left(V_{K}, \omega_{K}\right)+\sum_{K \in \mathcal{K}_{2}} V_{K} \mathcal{C}_{2}^{K, \text { mid }} \\
& +\mathbb{E}^{\mathbb{P}_{\sigma_{0}}}\left[I_{u}\left(h\left(S_{1}\right), V, S_{1}\right) e^{-u_{1}\left(S_{1}\right)}\right] .
\end{aligned}
$$

The gradients with respect to $V_{K}$ can also be written as

$$
\partial_{V_{K}} f^{K, \text { bid } / \text { ask }}\left(V_{K}, \omega_{K}\right)+\mathcal{C}_{2}^{K, \text { mid }}-\mathbb{E}^{\mathbb{P}_{\sigma_{0}}}\left[I_{K}\left(h\left(S_{1}\right), V, S_{1}\right) e^{-u_{1}\left(S_{1}\right)}\right] .
$$

And the hessians with respect to $V_{K_{1}}$ and $V_{K_{2}}$ are given by

$$
\delta_{K_{1}=K_{2}} \partial_{V_{K_{1}}^{2}}^{2} f^{K_{1}, \text { bid/ask }}\left(V_{K_{1}}, \omega_{K_{1}}\right)+\mathcal{C}_{2}^{K, \text { mid }}-\mathbb{E}^{\mathbb{P}}\left[\left(S_{2}-K_{1}\right)_{+}\left(S_{2}-K_{2}\right)_{+}\right] .
$$

With $\mathbb{P}:=e^{-\sum_{K \in \mathcal{K}_{2}} V_{K}\left(S_{2}-K\right)_{+}-h\left(S_{1}\right)\left(S_{2}-S_{1}\right)-u_{1}\left(S_{1}\right)} \mathbb{P}_{\sigma_{0}}$.

These formulas may be used to do the partial minimisation in $V$ with a "pure" Newton method, or with a quasi-Newton method which only requires the gradient.

IV Iterate steps (II)-(III) until convergence.

$\mathrm{V}$ Compute then the smile at $t_{2}$ for all $K \in \mathbb{R}_{+}$(this defines the marginal $\mathbb{P}^{2}$ ):

$$
\begin{aligned}
\mathbb{E}^{\mathbb{P}^{*}}\left[\left(S_{2}-K\right)_{+}\right] & =\mathbb{E}^{\mathbb{P}_{\sigma_{0}}}\left[I_{K}\left(h\left(S_{1}\right), V, S_{1}\right) e^{-u_{1}\left(S_{1}\right)}\right] \\
& \approx \mathbb{E}^{\mathbb{P}^{1} \mathcal{X}_{1}}\left[\frac{I_{K}\left(h\left(S_{1}\right), V, S_{1}\right)}{I_{u}\left(h\left(S_{1}\right), V, S_{1}\right)}\right] .
\end{aligned}
$$

\subsection{Convergence.}

Theorem 4.5 (Convergence rate). The map $\mathcal{G}_{12}$ reaches a minimum $\mathcal{G}_{12}^{*}$ at some $x^{*} \in \mathbb{R}^{2\left|\mathcal{X}_{1}\right|+\left|\mathcal{K}_{2}\right|}$ if and only if $\left(\mathbb{P}^{1},\left(\mathcal{C}_{2}^{K, \text { bid/ask }}\right)_{K \in \mathcal{K}_{2}}\right)$ is non-degenerate.

In this case, let $x_{0}=\left(u_{0}, h_{0}, V_{0}\right) \in \mathbb{R}^{2\left|\mathcal{X}_{1}\right|+\left|\mathcal{K}_{2}\right|}$, and for $n \geq 0$, let the $n^{\text {th }}$ iteration of the welldefined martingale Sinkhorn algorithm:

$$
\begin{aligned}
x_{n+1 / 2} & :=\left(u_{n}, h_{n}, V_{n+1}:=\underset{\psi}{\operatorname{argmin}} \mathcal{G}_{12}\left(u_{n}, h_{n}, \cdot\right)\right), \\
x_{n+1} & :=\left(u_{n+1}:=\underset{u}{\operatorname{argmin}} \mathcal{G}_{12}\left(\cdot, \cdot, V_{n+1}\right), h_{n+1}:=\underset{h}{\operatorname{argmin}} \mathcal{G}_{12}\left(\cdot, \cdot, V_{n+1}\right), V_{n+1}\right) .
\end{aligned}
$$


Then we may find $0<\lambda<1$, and $M>0$ such that

$$
\begin{aligned}
\mathcal{G}_{12}\left(x_{n}\right)-\mathcal{G}_{12}^{*} & \leq \quad \lambda^{n}\left(\mathcal{G}_{12}\left(x_{0}\right)-\mathcal{G}_{12}^{*}\right), \\
\left|x_{n}-x^{*}\right| \leq M \sqrt{\lambda}^{n} \quad \text { and } & \left|\nabla \mathcal{G}_{12}\left(x_{n}\right)\right| \leq M \sqrt{\lambda}^{n},
\end{aligned}
$$

for all $n \geq 0$.

The proof of Theorem 4.5 is reported to Section 5

Remark 4.6. Notice that

$$
\begin{aligned}
\nabla \mathcal{G} & =\sum_{s_{1} \in \mathcal{X}_{1}}\left(\mathbb{P}_{\mathcal{X}_{1}}^{1}\left[\left\{s_{1}\right\}\right]-\mathbb{P} \circ\left(S_{1}\right)^{-1}\left[\left\{s_{1}\right\}\right]\right) e_{s_{1}}+\sum_{s_{1} \in \mathcal{X}_{1}}\left(\mathbb{E}^{\mathbb{P}}\left[S_{2}-s_{1}, S_{1}=s_{1}\right]\right) e_{\left|\mathcal{X}_{1}\right|+s_{1}} \\
& +\sum_{i=1}^{k}\left(C_{K}-\mathbb{E}^{\mathbb{P}}\left[\left(S_{2}-K_{i}\right)_{+}\right]\right) e_{2\left|\mathcal{X}_{1}\right|+i}
\end{aligned}
$$

where $C_{K} \in\left[\mathcal{C}_{2}^{K \text {,bid }}, \mathcal{C}_{2}^{K \text {,ask }}\right]$, and $\left(e_{i}\right)_{1 \leq i \leq 2\left|\mathcal{X}_{1}\right|+\left|\mathcal{K}_{2}\right|}$ is the canonical basis. Therefore, this gradient is a crucial estimate of the mismatch of $\mathbb{P}$ in terms of first marginal, martingale property, and correctness of the call prices it gives.

Remark 4.7. We might obtain better stability and speed of convergence for the minimising of $\mathcal{G}$ by using an implied Newton minimization algorithm (see 3.3.5. in [9]). This algorithm consists of applying a truncated Newton algorithm on $\widetilde{\mathcal{G}}(V):=\min _{u_{1}, h_{1}} \mathcal{G}\left(u_{1}, h_{1}, V\right)$ which is strongly convex and smooth like $\mathcal{G}$, see Proposition 3.2 in [9]. This algorithm would have the same complexity, as we use a Newton algorithm of the same dimension $\left|\mathcal{K}_{2}\right|$ for the partial minimization in $V$ during phase (III) of the Sinkhorn algorithm, and the partial minimisation of $\mathcal{G}$ in $u$ and $h$ would be equivalent to steps (I) and (II). However, we can see from [9] that the convergence is much faster.

Remark 4.8. Even though the criterion from Definition 3.1 may not be easy to compute, trying to solve the entropic minimization reveals if a solution exists as otherwise the map $\mathcal{G}$ diverges to $-\infty$. In this case there is an arbitrage between the call prices and $\mathbb{P}^{1}$.

Remark 4.9. As we solve the problem building $\mathbb{P}^{i+1}$ after having built $\mathbb{P}^{i}$, we may encounter a situation in which the probability $\mathbb{P}^{1}$ may not be compatible with the call prices available in the intervals $\left[\mathcal{C}_{2}^{K, \text { bid }}, \mathcal{C}_{2}^{K \text {,ask }}\right]$. In this case we could solve a global entropic optimal transport problem, by projecting on all the times iteratively, like it is done in [5]. Another simpler solution would be to select in advance call prices in the bid-ask spreads that have no arbitrage. We have not encountered this situation in our numerical experiments using real market data.

4.4. Numerical examples. Below, we list some numerical examples involving numerous equity stocks/indices with various liquidity/maturities: Société Générale, Danone, Apple, SP500.

\section{Proofs of the Results.}

Lemma 5.1. Let $\left(u_{1}^{*}, h_{1}^{*}, V^{*}\right)$ be a minimiser of $\mathcal{G}_{12}$, then if we define

$$
\mathbb{P}^{*}\left(d s_{1}, d s_{2}\right):=m_{0}\left(d s_{1}, d s_{2}\right) e^{-\sum_{K \in \mathcal{K}_{2}} V_{K}^{*}\left(s_{2}-K\right)_{+}-u_{1}^{*}\left(s_{1}\right)-h_{1}^{*}\left(s_{1}\right)\left(s_{2}-s_{1}\right)},
$$



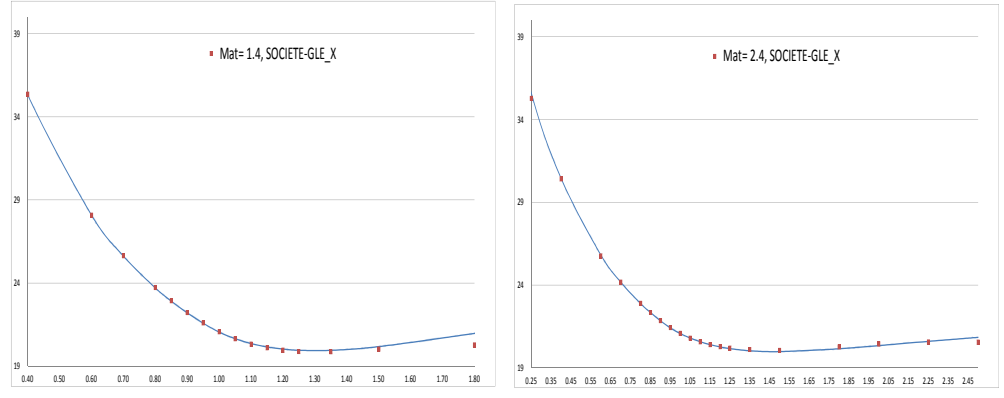

Figure 3. SOCIETE-GENERALE.
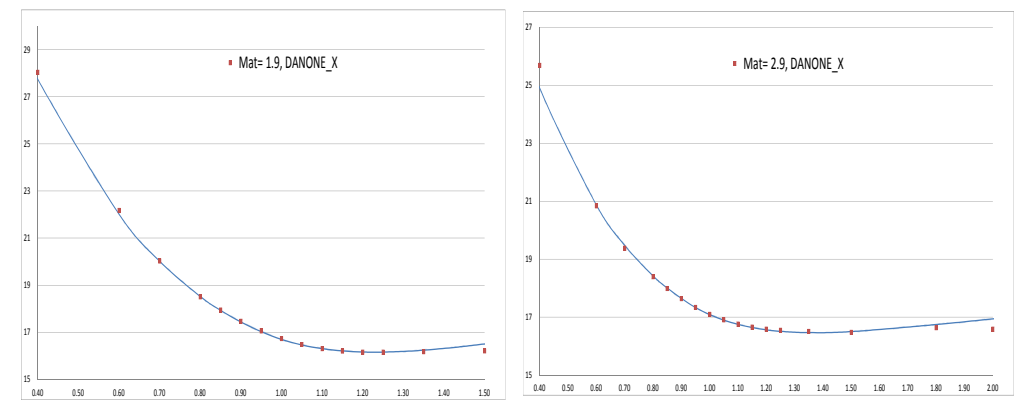

Figure 4. DANONE.
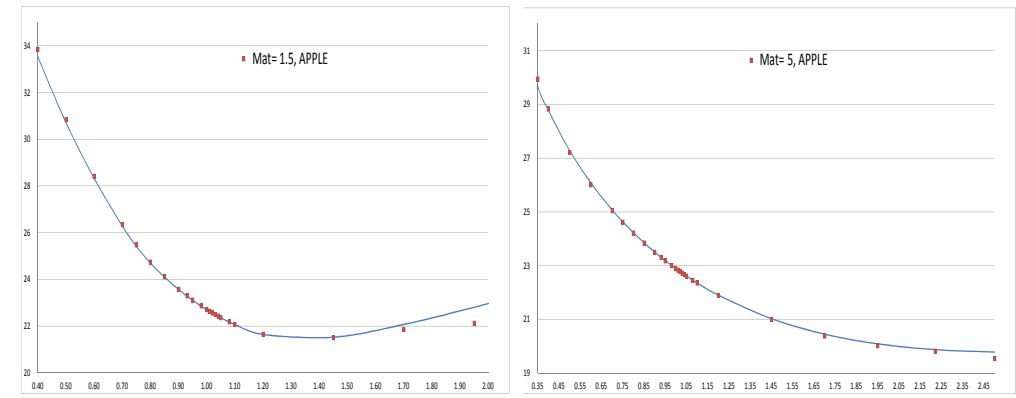

Figure 5. APPLE.

We have $\mathbb{P}^{*} \in \widetilde{\mathcal{M}}_{12}^{\text {Markov }}\left(\mathbb{P}^{1}\right)$.

Proof. The constraints are given by the Lagrange equations. For $s_{1} \in \mathcal{X}_{1}$, the fact that $\mathbb{P}^{*}\left(S_{1}=\right.$ $\left.s_{1}\right)=\mathbb{P}^{1}\left[s_{1}\right]$ is given by the equation $\partial_{u\left(s_{1}\right)} \mathcal{G}_{12}\left(u_{1}^{*}, h_{1}^{*}, V^{*}\right)$. The fact that $\mathbb{E}^{\mathbb{P}^{*}}\left[S_{2} \mid S_{1}=s_{1}\right]=s_{1}$ is given by the equation $\partial_{h\left(s_{1}\right)} \mathcal{G}_{12}\left(u_{1}^{*}, h_{1}^{*}, V^{*}\right)=0$. Finally, $\mathcal{C}_{2}^{K \text {,bid }} \leq \mathbb{E}^{\mathbb{P}^{*}}\left[\left(S_{2}-K\right)_{+}\right] \leq \mathcal{C}_{2}^{K \text {,ask }}$ is given by the equation $\partial_{V_{K}} \mathcal{G}_{12}\left(u_{1}^{*}, h_{1}^{*}, V^{*}\right)=0$.

Lemma 5.2. The map $\mathcal{G}_{12}$ reaches a minimum $\mathcal{G}_{12}^{*}$ at some $x^{*} \in \mathbb{R}^{2\left|\mathcal{X}_{1}\right|+\left|\mathcal{K}_{2}\right|}$ if and only if $\left(m_{0}, \mathbb{P}^{1},\left(\mathcal{C}_{2}^{K, \text { bid/ask }}\right)_{K \in \mathcal{K}_{2}}\right)$ is non-degenerate. 

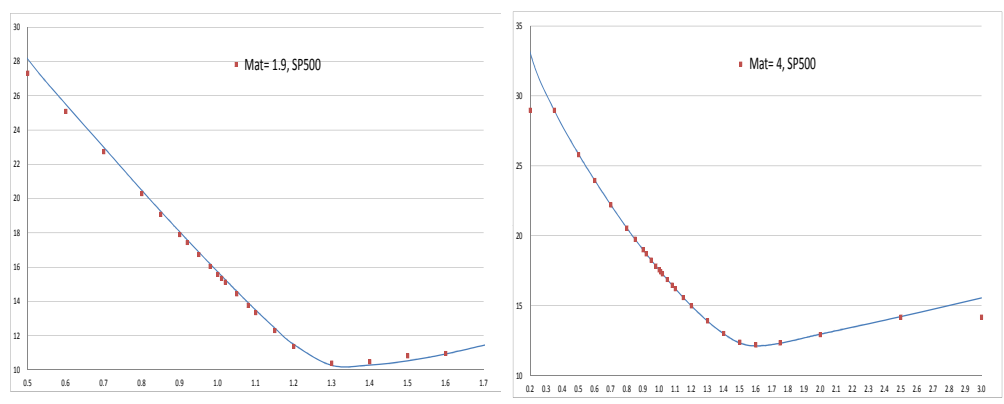

Figure 6. SP500.

Proof. For the sake of simplicity of notations, we denote $\mathcal{G}_{12}$ by $\mathcal{G}$.

Step 1: We assume that $\left(m_{0}, \mathbb{P}^{1},\left(\mathcal{C}_{2}^{K \text {,bid/ask }}\right)_{K \in \mathcal{K}_{2}}\right)$ is non-degenerate. Let $\mathcal{C} \in \mathbb{R}^{\left|\mathcal{K}_{2}\right|}$ be a valid call prices vector. Let us prove that $\mathcal{G}$ reaches a minimum at some $x^{*} \in \mathbb{R}^{2\left|\mathcal{X}_{1}\right|+\left|\mathcal{K}_{2}\right|}$. First we prove that $\lim _{|x| \rightarrow \infty} \mathcal{G}(x)=\infty$. Let $\left(x_{n}\right)_{n \geq 0} \subset \mathbb{R}^{2\left|\mathcal{X}_{1}\right|+\left|\mathcal{K}_{2}\right|}$ such that $\left|x_{n}\right| \longrightarrow \infty$. We assume for contradiction that up to replacing $x_{n}$ by a subsequence, $\mathcal{G}\left(x_{n}\right)$ is bounded from above by $A>0$. Then up to taking a subsequence of $\left(x_{n}\right)$, we may assume that $\frac{x_{n}}{\left|x_{n}\right|}$ converges to some $x \in \mathcal{U}:=\left\{x^{\prime} \in \mathbb{R}^{2\left|\mathcal{X}_{1}\right|+\left|\mathcal{K}_{2}\right|}\right\}$. Now let the random vector

$$
\Delta:=\left(\left(\delta_{S_{1}=s_{1}}\right)_{s_{1} \in \mathcal{X}_{1}},\left(\delta_{S_{1}=s_{1}}\left(S_{2}-S_{1}\right)\right)_{s_{1} \in \mathcal{X}_{1}},\left(\left(S_{2}-K\right)_{+}\right)_{K \in \mathcal{K}_{2}}\right),
$$

so that for $\left(\left(x_{1, s_{1}}\right)_{s_{1} \in \mathcal{X}_{1}},\left(x_{2, s_{1}}\right)_{s_{1} \in \mathcal{X}_{1}},\left(x_{3, K}\right)_{K \in \mathcal{K}_{2}}\right):=x \in \mathbb{R}^{2\left|\mathcal{X}_{1}\right|+\left|\mathcal{K}_{2}\right|}$, we have

$$
x \cdot \Delta=x_{1, S_{1}}+x_{2, S_{1}}\left(S_{2}-S_{1}\right)+\sum_{K \in \mathcal{K}_{2}} x_{3, K}\left(S_{2}-K\right)_{+} .
$$

and

$$
\mathcal{G}(x)=\sum_{s_{1} \in \mathcal{X}_{1}} x_{1, s_{1}} \mathbb{P}^{1}\left[s_{1}\right]+\sum_{K \in \mathcal{K}_{2}} f_{2}^{K, \text { bid } / \text { ask }}\left(x_{3, K}, \omega_{K}\right)+\sum_{K \in \mathcal{K}_{2}} x_{3, K} \mathcal{C}_{2}^{K, \text { mid }}+\int e^{-x \cdot \Delta} d m_{0} .
$$

Notice that as $\mathcal{C}_{2}^{K_{i} \text {, bid }} \leq \mathcal{C}_{i} \leq \mathcal{C}_{2}^{K_{i} \text {,ask }}$ for $1 \leq i \leq k$, we have that $\left(\mathcal{C}_{i}\right)_{1 \leq i \leq k}$ is the subgradient of $V \longmapsto \sum_{K \in \mathcal{K}_{2}} f_{2}^{K \text {,bid/ask }}\left(V_{K}, \omega_{K}\right)+\sum_{K \in \mathcal{K}_{2}} V_{K} \mathcal{C}_{2}^{K, \text { mid }}$ at some point $V^{0} \in \mathbb{R}^{\left|\mathcal{K}_{2}\right|}$. Then if we denote $b:=\sum_{K \in \mathcal{K}_{2}} f_{2}^{K, \text { bid } / \text { ask }}\left(V_{K}^{0}, \omega_{K}\right)+\sum_{K \in \mathcal{K}_{2}} V_{K}^{0} \mathcal{C}_{2}^{K, \text { mid }}$ and $a:=\left(\left(\mathbb{P}^{1}\left[s_{1}\right]\right)_{s_{1} \in \mathcal{X}_{1}}, 0,\left(\mathcal{C}_{i}\right)_{1 \leq i \leq k}\right)$, we have

$$
\mathcal{G}(x) \geq a \cdot x+b+\int e^{-x \cdot \Delta} d m_{0}
$$

Case 1: We may find $\left(s_{1}, s_{2}\right) \in \mathcal{X}_{1} \times \mathbb{R}_{+}$such that $x \cdot \Delta\left(s_{1}, s_{2}\right)<0$. As $x \cdot \Delta\left(s_{1}, \cdot\right)$ is affine by parts, we may find $\varepsilon>0$ and an open interval $s_{2} \in I \subset \mathbb{R}$ such that $x \cdot \Delta\left(s_{1}, \cdot\right) \leq-\varepsilon$ on $I$. Then for $x^{\prime}$ close enough to $x$, we have $x^{\prime} \cdot \Delta\left(s_{1}, \cdot\right) \leq-\frac{1}{2} \varepsilon$ on $I$. Then by (5.6), for $n$ large enough we have

$$
\begin{aligned}
\mathcal{G}\left(x_{n}\right) & \geq a \cdot x_{n}+b+\int e^{-x_{n} \cdot \Delta} d m_{0} \\
& \geq a \cdot x_{n}+b+m_{0}\left[\left\{y_{1}\right\} \times I\right] e^{\left|x_{n}\right| \frac{1}{2} \varepsilon} .
\end{aligned}
$$


Therefore, by the fact that $m_{0}\left[\left\{s_{1}\right\} \times I\right]>0$, we have that $\mathcal{G}\left(x_{n}\right)$ diverges to $\infty$ as $\left|x_{n}\right| \longrightarrow \infty$, a contradiction.

Case 2: $x \cdot \Delta \geq 0$ on $\mathcal{X}_{1} \times \mathbb{R}_{+}$. Then $\mathcal{G}\left(x_{n}\right) \geq a \cdot x_{n}+b=\left|x_{n}\right| a \cdot \frac{x_{n}}{\left|x_{n}\right|}+b$. As we assumed that $\mathcal{G}\left(x_{n}\right)$ is bounded and $\frac{x_{n}}{\left|x_{n}\right|}$ converges to $x$, we have

$$
a \cdot x \leq 0 .
$$

We denote $(u, h, V):=x$, identifying $u$ and $h$ as functions $\mathcal{X}_{1} \longrightarrow \mathbb{R}$, and we have

$$
x \cdot \Delta=u\left(S_{1}\right)+h\left(S_{1}\right)\left(S_{2}-S_{1}\right)+\sum_{K \in \mathcal{K}_{2}} V_{K}\left(S_{2}-K\right)_{+} .
$$

Let $\psi:=s \mapsto \sum_{K \in \mathcal{K}_{2}} V_{K}(s-K)_{+}$. We have $\psi\left(S_{2}\right) \geq-u\left(S_{1}\right)-h\left(S_{1}\right)\left(S_{2}-S_{1}\right)$. Then if we denote $f$, the convex hull of $\psi$ on $\mathbb{R}_{+}$, we have $\psi \geq f$ and for all $s_{1} \in \mathcal{X}_{1}$, we have $f\left(S_{2}\right) \geq$ $-u\left(s_{1}\right)-h\left(s_{1}\right) \cdot\left(S_{2}-s_{1}\right)$. Therefore, $f \geq-u$ from last functional inequality computed in $S_{2}=s_{1}$. By the fact that $f$ is the convex hull of $\psi$, which is piecewise affine, $f$ is also piecewise affine on the same intervals. Therefore, by using the notation $\mathcal{K}_{2}=\left(K_{i}\right)_{1 \leq i \leq k}$ from Definition 3.1. we may find $\lambda_{i} \geq 0$ for all $1 \leq i \leq k$ such that $f=s \mapsto f(0)+\nabla f(0) s+\sum_{i=1} \lambda_{i}\left(s-K_{i}\right)_{+}$. Recall that by Definition 3.1 we have that $\mathcal{C}_{0}=\mathbb{E}^{\mathbb{P}_{1}}\left[S_{1}\right]$, and $\mathcal{C}_{k+1}=1$, thus we have

$$
a \cdot x=\mathbb{E}^{\mathbb{P}^{1}}[u+f]+\sum_{i=0}^{k} \mu_{i} \mathcal{C}_{i}+\sum_{i=1}^{k+1} \lambda_{i}\left(\mathcal{C}_{i}-\mathbb{E}^{\mathbb{P}^{1}}\left[\left(S_{2}-K_{i}\right)_{+}\right]\right),
$$

where $\mu_{0}:=-\nabla f(0), \mu_{k+1}:=-f(0)$, and $\mu_{i}:=V_{K_{i}}-\lambda_{i}$ for $1 \leq i \leq k$ are the unique coefficients such that

$$
(\psi-f)\left(S_{2}\right)=\sum_{i=0}^{k} \mu_{i}\left(S_{2}-K_{i}\right)_{+} .
$$

Notice that $(\psi-f) \geq 0$, therefore for all $0 \leq i \leq k+1, \gamma_{i}:=(\psi-f)\left(K_{i+1}\right)=\left(M_{\text {call }}^{t} \mu\right)_{i} \geq 0$ by evaluating (5.8) in each $K_{i}$, where recall the definition of $M_{\text {call }}$ and the conventions on $i=k+1$ from Definition 3.1. Then $\mu=\left(M_{\text {call }}^{t}\right)^{-1} \gamma$, and $\mu \cdot \mathcal{C}=\left(M_{\text {call }}^{-1} \mathcal{C}\right) \cdot \gamma$. Finally,

$$
a \cdot x=\mathbb{E}^{\mathbb{P}^{1}}[u+f]+\left(M_{\text {call }}^{-1} \mathcal{C}\right) \cdot \gamma+\sum_{i=1}^{k} \lambda_{i}\left(\mathcal{C}_{i}-\mathbb{E}^{\mathbb{P}^{1}}\left[\left(S_{2}-K_{i}\right)_{+}\right]\right) .
$$

By (5.7), $a \cdot x$ is non-positive, therefore, the non-degeneracy of $\mathcal{C}$ gives that $\lambda_{1}=\ldots=\lambda_{k}=0, \gamma=0$, and $u+f=0$. Therefore $\mu=0, f=0, u=-f=0, V=0$. Therefore, $x \cdot \Delta=h\left(S_{1}\right)\left(S_{2}-S_{1}\right)$. By the fact that $x \cdot \Delta \geq 0, m_{0}-$ a.e., (vi) from Definition 3.1 implies that we have that $h=0$. Finally $x \cdot \Delta=0$ on $\mathcal{X}_{1} \times \mathbb{R}_{+}$, and finally $x=0$, which is a contradiction as $x \in \mathcal{U}$.

We proved that $\lim _{|x| \rightarrow \infty} \mathcal{G}(x)=\infty$. As $\mathcal{G}$ is convex, it reaches a minimum at some $x^{*} \in \mathbb{R}^{2\left|\mathcal{X}_{1}\right|+\left|\mathcal{K}_{2}\right|}$.

Step 2: Now we assume that $\mathcal{G}$ reaches a minimum. Let us denote $x^{*}$ this minimum and let $\mathbb{P}^{*}\left(d s_{1}, d s_{2}\right)=m_{0}\left(d s_{1}, d s_{2}\right) e^{-\sum_{K \in \mathcal{K}_{2}} V_{K}^{*}\left(s_{2}-K\right)_{+}-u_{1}^{*}\left(s_{1}\right)-h_{1}^{*}\left(s_{1}\right)\left(s_{2}-s_{1}\right)}$. By Proposition 4.1. we have that $\mathbb{P}^{*} \in \widetilde{\mathcal{M}}_{12}^{\text {Markov }}\left(\mathbb{P}^{1}\right)$. Notice also that the measure $\mathbb{P}^{*}$ is equivalent to the measure $m_{0}$. Therefore, for all $i$ the map $\theta_{i}:=\left(S_{2}-K_{i}\right)_{+}-\left(S_{1}-K_{i}\right)_{+}-\mathbf{1}_{S_{1} \geq K_{i}}\left(S_{2}-S_{1}\right)$ is non-negative and non-(zero $\mathbb{P}^{*}$-a.e.). Therefore $\mathbb{E}^{\mathbb{P}^{*}}\left[\theta_{i}\right]>0$. Finally we observe that if we denote $\mathcal{C}_{i}:=\mathbb{E}^{\mathbb{P}^{*}}\left[\left(S_{2}-K\right)_{+}\right]$, then we have $\mathbb{E}^{\mathbb{P}^{*}}\left[\theta_{i}\right]=\mathcal{C}_{i}-\mathbb{E}^{\mathbb{P}^{1}}\left[\left(S_{1}-K\right)_{+}\right]$from the martingale property of $\mathbb{P}^{*}$, and $\mathcal{C}_{2}^{K_{i} \text {, bid }} \leq \mathcal{C}_{i} \leq \mathcal{C}_{2}^{K_{i} \text {,ask }}$ 
as $\mathbb{P}^{*} \in \widetilde{\mathcal{M}}_{12}^{\text {Markov }}\left(\mathbb{P}^{1}\right)$. Now for $1 \leq i \leq k$, let $f_{i}$ the piecewise affine map such that $f$ is zero on $\left[0, K_{i-1}\right]$, with $f\left(K_{i}\right)=1$, affine on $\left[K_{i-1}, K_{i}\right],\left[K_{i}, K_{i+1}\right]$, and $\left[K_{i+1}, \infty\right)$, if $i \neq k$, and $f_{k}$ is constant equal to 1 on $\left[K_{k}, \infty\right]$. We observe that for all $i, f_{i}$ is non-negative and non-zero $\mathbb{P}^{*}$-a.e. Furthermore, $0<\mathbb{E}^{\mathbb{P}^{*}}\left[f_{i}\right]=\left(M_{\text {call }}^{-1} \mathcal{C}\right)_{i}$. We proved that $\left(m_{0}, \mathbb{P}^{1},\left(\mathcal{C}_{2}^{K \text {,bid/ask }}\right)_{K \in \mathcal{K}_{2}}\right)$ is non-degenerate as the other properties are obvious.

Proof of Proposition 4.1 By introducing dual variables $u^{\text {bid }}, u^{\text {ask }} \in\left(\mathbb{R}_{+}\right)^{\mathcal{K}_{2}}$ for the inequalities for the call prices at bid and at ask, inf $\mathcal{G}_{12}$ may be written as

$$
\begin{aligned}
\inf \mathcal{G}_{12}= & -\operatorname{unf}_{u^{\text {bid }}, u^{\text {ask }} \in\left(\mathbb{R}_{+}\right)^{\mathcal{K}_{2}, v_{K} \in \mathbb{R}, u_{1}, h_{1} \in \mathbb{R}^{\mathcal{X}_{1}}}} \mathbb{E}^{\mathbb{P}^{1}}\left[u_{1}\left(S_{1}\right)\right]+\sum_{K \in \mathcal{K}_{1}} u_{K}^{\text {ask }} \mathcal{C}_{K}^{\text {ask }}-u_{K}^{\text {bid }} \mathcal{C}_{K}^{\text {bid }}+v_{K} \mathcal{C}_{K}^{\text {mid }} \\
& +\frac{1}{2} v_{K}^{2} \omega_{K}+\mathbb{E}^{m_{0}}\left[e^{-\sum_{K \in \mathcal{K}}\left(u_{K}^{\text {ask }}-u_{K}^{\text {bid }}+v_{K}\right)\left(S_{1}-K\right)_{+}-u_{1}\left(S_{1}\right)-h_{1}\left(S_{1}\right)\left(S_{2}-S_{1}\right)}\right] .
\end{aligned}
$$

By setting $v:=V-u^{\text {ask }}+u^{\text {bid }}$, the function, to be minimized, is equivalent to

$$
\begin{aligned}
& \mathbb{E}^{\mathbb{P}^{1}}\left[u_{1}\left(S_{1}\right)\right]+\sum_{K \in \mathcal{K}_{1}} u_{K}^{\text {ask }}\left(\mathcal{C}_{1}^{K, \text { ask }}-\mathcal{C}_{1}^{K, \text { mid }}\right)-u_{K}^{\text {bid }}\left(\mathcal{C}_{1}^{K, \text { bid }}-\mathcal{C}_{1}^{K, \text { mid }}\right)+V_{K} \mathcal{C}_{1}^{K, \text { mid }} \\
+ & \frac{1}{2}\left(V-u^{\text {ask }}+u^{\text {bid }}\right)^{2} \cdot \omega+\mathbb{E}^{m_{0}}\left[e^{-\sum_{K \in \mathcal{K}} V_{K}\left(S_{1}-K\right)_{+}-u_{1}\left(S_{1}\right)-h_{1}\left(S_{1}\right)\left(S_{2}-S_{1}\right)}\right] .
\end{aligned}
$$

We observe that the minimization over $u^{\text {ask }}$ and $u^{\text {bid }}$ can be exactly performed and we obtain finally an unconstrained optimization over $V$.

By Lemma 5.2 the non degeneracy of $\left(m_{0}, \mathbb{P}^{1},\left(\mathcal{C}_{2}^{K, \text { bid/ask }}\right)_{K \in \mathcal{K}_{2}}\right)$ implies that $\mathcal{G}_{12}$ reaches a minimum. Let $\mathbb{P}^{*} \in \widetilde{\mathcal{M}}_{12}^{\text {Markov }}\left(\mathbb{P}^{1}\right)$ from Lemma $5.1 \mathbb{P}^{*}$ is the optimiser of 4.3 from Proposition 1 in $[2]$.

Proof of Proposition 3.3 The proof is the same than the one from Proposition 4.1, taking $\left(m_{0}, \delta_{S_{0}},\left(\mathcal{C}_{1}^{K, \text { bid/ask }}\right)_{K \in \mathcal{K}_{1}}\right)$ instead of $\left(m_{0}, \mathbb{P}^{1},\left(\mathcal{C}_{2}^{K, \text { bid } / \text { ask }}\right)_{K \in \mathcal{K}_{2}}\right)$.

Proof of Theorem 4.5 For the sake of simplicity of notations, we denote $\mathcal{G}_{12}$ by $\mathcal{G}$. The first equivalence is given by Lemma 5.2

Step 1: The convergence result stems from an indirect application of Theorem 5.2 in [4. By a direct application of this theorem we get that

$$
\mathcal{G}\left(x_{k}\right)-\mathcal{G}\left(x^{*}\right) \leq\left(1-\frac{\sigma}{\min \left(L_{1}, L_{2}\right)}\right)^{n-1}\left(\mathcal{G}\left(x_{0}\right)-\mathcal{G}\left(x^{*}\right)\right),
$$

with $L_{1}$ (resp. $L_{2}$ ) is the Lipschitz constant of the $V$-gradient (resp. $(u, h)$-gradient) of $\mathcal{G}$, and $\sigma$ is the strong convexity parameter of $\mathcal{G}$. Furthermore, the strong convexity gives that

$$
\left|x_{k}-x^{*}\right| \leq \sqrt{\frac{2}{\sigma}}\left(\mathcal{G}\left(x_{k}\right)-\mathcal{G}\left(x^{*}\right)\right)^{\frac{1}{2}}
$$

Finally, by definition of $L_{1}$ and $L_{2}$, we have

$$
\left|\nabla \mathcal{G}\left(x_{k}\right)\right| \leq\left(L_{1}+L_{2}\right)\left|x_{k}-x^{*}\right| .
$$


These inequalities would prove the theorem with

$$
\lambda=1-\frac{\sigma}{\min \left(L_{1}, L_{2}\right)} \quad \text { and } \quad M=\left(L_{1}+L_{2}+1\right) \sqrt{\frac{2}{\sigma}} .
$$

However the gradient $\nabla \mathcal{G}$ is locally but not globally Lipschitz, nor $\mathcal{G}$ strongly convex. Therefore we need to refine the theorem by looking carefully at where these constants are used in its proof.

Step 2: The constant $L_{1}$ is used for Lemma 5.1 in [4. We need for all $k \geq 0$ to have $\mathcal{G}\left(x_{k}\right)-$ $\overline{\mathcal{G}\left(x_{k+1 / 2}\right)} \geq \frac{1}{2 L_{1}}\left|\nabla \mathcal{G}\left(x_{k}\right)\right|^{2}$. We want to find $C, L>0$ such that $\mathcal{G}\left(x_{k}\right)-\mathcal{G}\left(x_{k}-C \nabla \mathcal{G}\left(x_{k}\right)\right) \geq$ $\frac{1}{2 L}\left|\nabla \mathcal{G}\left(x_{k}\right)\right|$, then $L$ may be use to replace $L_{1}$ in the final step of the proof of Lemma 5.1 in [4]. By the fact that $\lim _{|x| \rightarrow \infty} \mathcal{G}(x)=\infty$, the set $\mathfrak{C}\left(x_{0}\right):=\left\{x \in \mathbb{R}^{2\left|\mathcal{X}_{1}\right|+\left|\mathcal{K}_{2}\right|}: \mathcal{G}(x) \leq \mathcal{G}\left(x_{0}\right)\right\}$ is compact. Then $\nabla \mathcal{G}$ is bounded on $\mathfrak{C}\left(x_{0}\right)$. Therefore we may find $M_{1}>0$ such that for all $k$, we have $\mid \nabla \mathcal{G}\left(x_{k}\right)$. $\Delta \mid \leq M_{1}\left(1+\left|S_{2}\right|\right)$. Furthermore, let $F(C):=\sup _{(u, x) \in \mathcal{U} \times \mathfrak{C}\left(x_{0}\right)} \int_{0}^{1} \int(u \cdot \Delta) e^{-x \cdot \Delta} e^{t C M_{1}\left(1+\left|S_{2}\right|\right)} d m_{0} d t$. We have

$$
\begin{aligned}
\mathcal{G}\left(x_{k}\right)-\mathcal{G}\left(x_{k}-C \nabla \mathcal{G}\left(x_{k}\right), h_{k}\right)= & \nabla \mathcal{G}\left(x_{k}\right) \cdot\left(-C \nabla \mathcal{G}\left(x_{k}\right)\right) \\
& -C^{2} \int_{0}^{1}(1-t) D^{2} \mathcal{G}\left(x_{k}-t C \nabla \mathcal{G}\left(x_{k}\right)\right)\left(\nabla \mathcal{G}\left(x_{k}\right)\right)^{2} d t \\
= & C\left|\nabla \mathcal{G}\left(x_{k}\right)\right|^{2} \\
& -C^{2} \int_{0}^{1}(1-t) \int\left(\nabla \mathcal{G}\left(x_{k}\right) \cdot \Delta\right)^{2} e^{-x_{k} \cdot \Delta} e^{t C \nabla \mathcal{G}\left(x_{k}\right) \cdot \Delta} d m_{0} d t \\
\geq & C\left|\nabla \mathcal{G}\left(x_{k}\right)\right|^{2}-C^{2}\left|\nabla \mathcal{G}\left(x_{k}\right)\right|^{2} F(C) \\
= & \left(C-C^{2} F(C)\right)\left|\nabla \mathcal{G}\left(x_{k}\right)\right|^{2} .
\end{aligned}
$$

As $F$ is non-decreasing finite, then when $C \longrightarrow 0$ we have $\frac{C-C^{2} F(C)}{C} \longrightarrow 1$. Then for $C$ small enough, let $L:=\frac{1}{2\left(C-C^{2} F(C)\right)}>0$. We get

$$
\mathcal{G}\left(x_{k}\right)-\mathcal{G}\left(x_{k}-C \nabla \mathcal{G}\left(x_{k}\right)\right) \geq \frac{1}{2 L}\left|\nabla \mathcal{G}\left(x_{k}\right)\right|^{2} .
$$

Step 3: The constant $\sigma$ is used to get the result from (3.21) in [4]. Then we just need the inequality

$$
\mathcal{G}(y) \geq \mathcal{G}(x)+\nabla \mathcal{G}(x) \cdot(y-x)+\frac{\sigma}{2}|y-x|^{2},
$$

to hold for some $y=x^{*}$ and $x=x_{k}$ for all $k \geq 0$. Now we give a lower bound for $\sigma$. The map $(u, x) \longmapsto D^{2} \mathcal{G}(x) u^{2}=\int(u \cdot \Delta)^{2} e^{-x \cdot \Delta} d m_{0}>0$ is continuous on $\mathcal{U} \times \mathfrak{C}\left(x_{0}\right)$ compact, therefore it has a lower bound $\sigma>0$. This constant also works for 5.10 . Similar, $\sup _{(u, x) \in \mathcal{U} \times \mathfrak{C}\left(x_{0}\right)} D^{2} \mathcal{G}(x) u^{2}$ may replace $L_{1}+L_{2}$ from 5.11 .

Step 4: Finally, as we focus on the $L_{1}$ optimization phase, we may replace $n-1$ by $n$ in the convergence formula (5.9), see the proof of Theorem 5.2 in [4].

Now the existence of $M>0$ stems from the facts that $\mathcal{G}\left(x_{k}\right)-\mathcal{G}\left(x^{*}\right) \geq \frac{1}{2} \sigma\left|x_{k}-x^{*}\right|^{2}$, and $\left|\nabla \mathcal{G}\left(x_{k}\right)\right| \leq L\left|x_{k}-x^{*}\right|$

The result is proved. 


\section{REFERENCES}

[1] Jesper Andreasen and Brian Huge. Expanded forward volatility. Risk, 26(1):101, 2013.

[2] M Avellaneda. Minimum entropy calibration of asset pricing models, internat. J. Theoret. Appl. Finance, 1:447472, 1998.

[3] Marco Avellaneda, Robert Buff, Craig Friedman, Nicolas Grandechamp, Lukasz Kruk, and Joshua Newman. Weighted monte carlo: a new technique for calibrating asset-pricing models. International Journal of Theoretical and Applied Finance, 4(01):91-119, 2001.

[4] Amir Beck and Luba Tetruashvili. On the convergence of block coordinate descent type methods. SIAM journal on Optimization, 23(4):2037-2060, 2013

[5] Jean-David Benamou, Guillaume Carlier, Marco Cuturi, Luca Nenna, and Gabriel Peyré. Iterative bregman projections for regularized transportation problems. SIAM Journal on Scientific Computing, 37(2):A1111A1138, 2015.

[6] Peter Carr and Dilip B Madan. A note on sufficient conditions for no arbitrage. Finance Research Letters, 2(3):125-130, 2005.

[7] Laurent Cousot. Conditions on option prices for absence of arbitrage and exact calibration. Journal of Banking E Finance, 31(11):3377-3397, 2007.

[8] Marco Cuturi. Sinkhorn distances: Lightspeed computation of optimal transport. In Advances in neural information processing systems, pages 2292-2300, 2013.

[9] Hadrien De March. Entropic resolution for multi-dimensional optimal transport. arXiv preprint arXiv:1812.11104, 2018.

[10] Bruno Dupire. Pricing and hedging with smiles. Mathematics of derivative securities, 1(1):103-111, 1997.

[11] Peter K Friz, Jim Gatheral, Archil Gulisashvili, Antoine Jacquier, and Josef Teichmann. Large deviations and asymptotic methods in finance, volume 110. Springer, 2015.

[12] Jim Gatheral and Antoine Jacquier. Convergence of heston to svi. Quantitative Finance, 11(8):1129-1132, 2011.

[13] Jim Gatheral and Antoine Jacquier. Arbitrage-free svi volatility surfaces. Quantitative Finance, 14(1):59-71, 2014.

[14] Gaoyue Guo and Jan Obloj. Computational methods for martingale optimal transport problems. arXiv preprint arXiv:1710.07911, 2017.

[15] Pierre Henry-Labordère. Analysis, geometry, and modeling in finance: Advanced methods in option pricing. Chapman and Hall/CRC, 2008.

[16] Pierre Henry-Labordere. Calibration of local stochastic volatility models to market smiles: A monte-carlo approach. 2009.

[17] Pierre Henry-Labordere and Nizar Touzi. An explicit martingale version of brenier's theorem. 2013.

[18] Sigrid Källblad, Xiaolu Tan, Nizar Touzi, et al. Optimal skorokhod embedding given full marginals and azémayor peacocks. The Annals of Applied Probability, 27(2):686-719, 2017.

[19] Alex Lipton and Artur Sepp. Filling the gaps. 2011.

[20] Alexander Lipton. Masterclass with deutsche bank. the vol smile problem. RISK-LONDON-RISK MAGAZINE LIMITED-, 15(2):61-66, 2002.

[21] Quentin Mérigot. A multiscale approach to optimal transport. In Computer Graphics Forum, volume 30, pages 1583-1592. Wiley Online Library, 2011.

[22] Gabriel Peyré, Marco Cuturi, et al. Computational optimal transport. Technical report, 2017.

[23] Bernhard Schmitzer. Stabilized sparse scaling algorithms for entropy regularized transport problems. arXiv preprint arXiv:1610.06519, 2016.

[24] Richard Sinkhorn and Paul Knopp. Concerning nonnegative matrices and doubly stochastic matrices. Pacific Journal of Mathematics, 21(2):343-348, 1967.

[25] Volker Strassen. The existence of probability measures with given marginals. The Annals of Mathematical Statistics, pages 423-439, 1965. 